TRANSACTIONS OF THE

AMERICAN MATHEMATICAL SOCIETY

Volume 362, Number 7, July 2010, Pages 3841-3881

S 0002-9947(10)04974-3

Article electronically published on February 24, 2010

\title{
DENSITY RESULTS FOR AUTOMORPHIC FORMS ON HILBERT MODULAR GROUPS II
}

\author{
ROELOF W. BRUGGEMAN AND ROBERTO J. MIATELLO
}

\begin{abstract}
We obtain an asymptotic formula for a weighted sum over cuspidal eigenvalues in a specific region, for $\mathrm{SL}_{2}$ over a totally real number field $F$, with a discrete subgroup of Hecke type $\Gamma_{0}(I)$ for a non-zero ideal $I$ in the ring of integers of $F$. The weights are products of Fourier coefficients. This implies in particular the existence of infinitely many cuspidal automorphic representations with multi-eigenvalues in various regions growing to infinity. For instance, in the quadratic case, the regions include floating boxes, floating balls, sectors, slanted strips (see $\S 1.2 .4-1.2 .13$ ) and products of prescribed small intervals for all but one of the infinite places of $F$. The main tool in the derivation is a sum formula of Kuznetsov type (Sum formula for $\mathrm{SL}_{2}$ over a totally real number field, Theorem 2.1).
\end{abstract}

\section{Contents}

Introduction 3841

1. Preliminaries and discussion of main results 3844

2. Sum formula 3852

3. Upper bound 3861

4. Asymptotic formula, first stage 3863

5. Asymptotic formula, second stage 3871

6. Special families 3874

References 3880

\section{INTRODUCTION}

Let $F$ be a totally real number field of dimension $d$, and let $\mathcal{O}_{F}$ be its ring of integers. If $I$ is a non-zero ideal in $\mathcal{O}_{F}$, let $\Gamma=\Gamma_{0}(I)$ denote the congruence subgroup of Hecke type of the Hilbert modular group. We allow a character of $\Gamma_{0}(I)$ of the form $\left(\begin{array}{ll}a & b \\ c & d\end{array}\right) \mapsto \chi(d)$, with $\chi$ a character modulo $I$.

The goal of the present paper is to obtain distribution results for cuspidal automorphic representations of $G \cong \operatorname{SL}(2, \mathbf{R})^{d}$ with eigenvalue parameters in a subset $\Omega_{t}$ of the multi-eigenvalue space, as $t \rightarrow \infty$, under some general conditions on the family $\Omega_{t}$.

Received by the editors October 17, 2008.

2000 Mathematics Subject Classification. Primary 11F30, 11F41, 11F72, 22E30.

(C)2010 American Mathematical Society

Reverts to public domain 28 years from publication 
Let $V_{\varpi}$ be a cuspidal automorphic representation, with elements transforming under the above character of $\Gamma_{0}(I)$ and with a compatible central character. The Fourier coefficients of automorphic forms in $V_{\varpi}$ can be normalized so that they are independent of the chosen automorphic form in $V_{\varpi}$. This results in coefficients $c^{r}(\varpi)$ describing the Fourier expansion at the cusp $\infty$. The Fourier term order $r$ runs through the inverse different $\mathcal{O}^{\prime}$ of $F$.

We denote by $\lambda_{\varpi}=\left(\lambda_{\varpi, j}\right)_{j} \in \mathbb{R}^{d}$ the vector of eigenvalues of the Casimir operators at the infinite (real) places of $F$. For compact sets $\Omega \subset \mathbb{R}^{d}$, we consider the counting functions

$$
\mathrm{N}^{r}(\Omega):=\mathrm{N}_{\xi, \chi}^{r}(\Omega):=\sum_{\varpi, \lambda_{\varpi} \in \Omega}\left|c^{r}(\varpi)\right|^{2} .
$$

The representations $\varpi$ run through an orthogonal system of irreducible subspaces of $L_{\xi}^{2, \text { cusp }}\left(\Gamma_{0}(I) \backslash G, \chi\right)$, for a fixed choice of the character $\chi$ of $\Gamma_{0}(I)$ and of the central character (determined by $\xi \in\{0,1\}^{d}$ ).

The main result in this paper asserts that if the family $t \mapsto \Omega_{t}$ satisfies certain mild conditions, then

$$
\mathrm{N}^{r}\left(\Omega_{t}\right)=\frac{2 \sqrt{\left|D_{F}\right|}}{(2 \pi)^{d}} \operatorname{Pl}\left(\Omega_{t}\right)+o\left(V_{1}\left(\Omega_{t}\right)\right) \quad(t \rightarrow \infty)
$$

for all non-zero $r \in \mathcal{O}^{\prime}$. By $D_{F}$ we denote the discriminant of $F$ over $\mathbb{Q}$, and by $\mathrm{Pl}$ the Plancherel measure of $G$. The error term contains a reference measure $V_{1}$ which, under some general assumptions, is comparable to $\mathrm{Pl}$.

Roughly speaking, we show that the asymptotic formula (2) holds for the family $t \mapsto \Omega_{t}$ under the conditions that $\Omega_{t}$ grows in at least one coordinate direction, and that the boundary $\partial \Omega_{t}$ is small in comparison with $\Omega_{t}$ itself. On the other hand, it is often convenient to use, instead of $\lambda \in \mathbb{R}^{d}$, the corresponding spectral parameter $\nu_{\varpi} \in([0, \infty) \cup i(0, \infty))^{d}$. We use a tilde to indicate that the relevant measures like $\tilde{\mathrm{N}}^{r}, \widetilde{\mathrm{Pl}}$ and $\tilde{V}_{1}$ are taken in the variable $\nu$, and we write

$$
\tilde{\mathrm{N}}^{r}\left(\tilde{\Omega}_{t}\right)=\sum_{\varpi, \nu_{\varpi} \in \tilde{\Omega}_{t}}\left|c^{r}(\varpi)\right|^{2}
$$

with $\nu_{\varpi, j} \in[0, \infty) \cup i(0, \infty)$ such that $\lambda_{\varpi, j}=\frac{1}{4}-\nu_{\varpi, j}^{2}$.

In Theorems 4.6 and 5.3 we prove asymptotic statements in terms of the quantities $\mathrm{N}^{r}\left(\Omega_{t}\right)$ and $\tilde{\mathrm{N}}^{r}\left(\tilde{\Omega}_{t}\right)$, respectively, and this enables us to show occurrence and density of representations for a wide class of families of sets $t \mapsto \Omega_{t}$. For illustration, we now list some of the distribution results that are obtained in the quadratic case.

(i) Small rectangles. Let $d=2$. Let $[\alpha, \beta] \subset[1 / 4, \infty)$ and consider for $t$ large $\Omega_{t}=[\alpha, \beta] \times[t, t+\sqrt{t}]$. Formula (2) implies

$$
\mathrm{N}^{r}\left(\Omega_{t}\right) \sim \frac{\sqrt{D_{F}}}{2 \pi^{2}} \int_{\alpha}^{\beta} \tanh \pi \sqrt{\lambda-\frac{1}{4}} d \lambda t^{1 / 2} \quad(t \rightarrow \infty) .
$$

In particular, there are infinitely many $\varpi$ with $\lambda_{\varpi, 1} \in[\alpha, \beta]$. These $\varpi$ have a second component of unitary principal series type. A similar result holds with $\varpi_{2}$ of discrete series type.

On the other hand if $[\alpha, \beta] \subset\left[\lambda_{0}, \frac{1}{4}\right)$ and $\Omega_{t}=[\alpha, \beta] \times[t, \sqrt{t}]$, then

$$
\mathrm{N}^{r}\left(\Omega_{t}\right)=o\left(t^{1 / 2}\right) \quad(t \rightarrow \infty) .
$$


This gives an upper bound for the weighted density of $\varpi_{1}$ of complementary series type.

As another example, if one takes $\Omega_{t}=[t, t+\sqrt{t}] \times[c t, c t+\sqrt{t}]$, with $t$ large and $c \geq 1$, then

$$
\mathrm{N}^{r}\left(\Omega_{t}\right) \sim C_{c} t \quad(t \rightarrow \infty) .
$$

(ii) Slanting strips. Let $d=2$, and put, in terms of the spectral parameter,

$$
\tilde{\Omega}_{t}=\left\{\left(\nu_{1}, \nu_{2}\right) \in(i[1, \infty))^{2}: t \leq\left|\nu_{1}\right| \leq 2 t, a\left|\nu_{1}\right|+b \leq\left|\nu_{2}\right| \leq a\left|\nu_{1}\right|+c\right\},
$$

with $a>0, c>b$ fixed and $t$ large. Then

$$
\tilde{\mathrm{N}}^{r}\left(\tilde{\Omega}_{t}\right) \sim C_{a, b, c} t^{3} \quad(t \rightarrow \infty) .
$$

This shows that we see infinitely many points $\nu_{\varpi}$ in a slanted direction. We note that this slanting strip becomes a sector in $\lambda$-space.

(iii) Sectors. Let $d=2$, and fix $0<p<q, \alpha>\frac{1}{2}$. For $t$ large put

$$
\Omega_{t}=\left\{\left(\lambda_{1}, \lambda_{2}\right) \in[0, \infty)^{2}: t \leq \lambda_{1} \leq t+t^{\alpha}, p \lambda_{1} \leq \lambda_{2} \leq q \lambda_{1}\right\} .
$$

Then we have

$$
\mathrm{N}^{r}\left(\Omega_{t}\right) \sim C_{p, q} t^{1+\alpha} \quad(t \rightarrow \infty) .
$$

(iv) Spaces of holomorphic cusp forms. Take $\tilde{\Omega}_{b}$ equal to the singleton $b=$ $\left(b_{1}, \ldots, b_{j}\right) \in \mathbb{Z}^{d}$ with $b_{j} \geq 1$ and agreeing with the central character, i.e., $b_{j} \equiv \xi_{j} \bmod 2$ for $1 \leq j \leq d$. These are the weights for which there may be holomorphic cusp forms on the product of $d$ copies of the upper half-plane for the character $\chi$ of $\Gamma_{0}(I)$. Corollary 4.2 shows that for some positive constant $C$ we have

$$
\tilde{\mathrm{N}}^{r}\left(\tilde{\Omega}_{b}\right) \sim C \prod_{j=1}^{d} \frac{b_{j}-1}{2} \quad \text { as } \prod_{j=1}^{d} \frac{b_{j}-1}{2} \rightarrow \infty .
$$

If we take $r$ totally positive, then the quantity $\tilde{\mathrm{N}}^{r}\left(\tilde{\Omega}_{b}\right)$ has an expression in terms of Fourier coefficients of holomorphic cusp forms. In particular, this implies that there are only finitely many weights $b$ with $b_{j} \geq 2$ for all $j$ for which the corresponding space of cusp forms is non-zero. (We do not obtain information concerning weights $b$ for which $b_{j}=1$ for some $j$.)

We will give a much more complete list of applications of the main asymptotic formula in 1.2 .41 .2 .13

By using the Selberg trace formula (21, 8]), some unweighted distribution results related to those in this paper have been obtained in [9] and [10, while results connected with Weyl's laws in different contexts have been proved by several authors, e.g., [6], [5, [7, [19, [18, [14, [20, 17] and [16].

The main tool for the results in the present paper is the Kuznetsov type sum formula in Theorem 3.21 of [3, which we recall in 2.1 . It leads to sums weighted by a product of Fourier coefficients. The results obtained here may be viewed as a generalization of the results in 2. To explain the difference, we note that the sum formula gives a linear relation between four terms. The two main terms in the present context are a weighted sum of a test function $\varphi$ over the $\nu_{\varpi}$ and an integral of $\varphi$ against the Plancherel measure. The test function has a product form $\varphi(\nu)=\prod_{j} \varphi_{j}\left(\nu_{j}\right)$, where $j$ runs over the infinite places of the totally real number 
field $F$. For the terms that are principal in this paper this product form is nonessential. However to show that the other terms are small we need, for one of those terms (the sum of Kloosterman sums), estimates of a Bessel transformation of each of the factors $\varphi_{j}$. This forces the product structure upon us, in contrast with the case of the Selberg trace formula. There the integral transformation is the Fourier transform, which respects rotations.

In 2] we chose each factor $\varphi_{j}$ as a Gaussian kernel. For the places in a non-empty set $Q$ of real places, this kernel was an approximation of the constant function one, and for the other places it was chosen as an approximation of a delta distribution. This led us to asymptotic results for regions $\Omega_{X}$ of the form

$$
\prod_{j \notin Q}\left[a_{j}, b_{j}\right] \times \prod_{j \in Q}\left\{\left(\lambda_{j}\right)_{j \in Q}: \sum_{j \in Q}\left|\lambda_{j}\right| \leq X\right\} .
$$

The purpose of this paper is to work with sets having a much more general form in the coordinates in $Q$. To do this, the test functions have to be chosen in a much more complicated way. Our choice is indicated in Lemma 2.2. The idea is to take, for each place in $Q$, a Gaussian kernel of moderate sharpness. We approximate the characteristic function of sets in $\prod_{j \in Q}(\mathbb{R} \cup i \mathbb{R})$ by a convolution with this Gaussian kernel. At the real places outside $Q$ we take a general test function to be specified at a later stage. An application of the sum formula gives the relation in Proposition 2.4. The use of a Tauberian argument as in 22 is no longer possible. To be able to handle the error terms, we first give in $\$ 3$ an upper bound for the weighted sums under consideration. After that we adapt the sharpness of the test functions to the family of sets we consider.

This leads to Theorem 4.6 where we lose control over the size of the error term and have to be content with an asymptotic result. This is so because the size of the error term depends on the family of sets in a complicated way, as equations (94) - (96) show, and the error term is almost as large as the main term. The second stage of the method, in $\$ 5$, involves choosing the factors of the test function at the real places outside $Q$ as approximations of characteristic functions of intervals in the coordinate $\lambda_{j}$. The central result is Theorem [5.3. It is specialized in $\$ 6$ to various families of sets that include those mentioned above.

The sum formula involves products $\overline{c^{r}(\varpi)} c^{r^{\prime}}(\varpi)$ for two non-zero Fourier terms orders $r$ and $r^{\prime}$. Its application in the present paper works well if $\frac{r^{\prime}}{r}$ is totally positive. We intend to apply the asymptotic results, under this assumption, in subsequent work where we will take eigenvalues of Hecke operators into account.

\section{Preliminaries and Discussion of Main Results}

This section serves to recall results and fix notation, and after that to state the main results of this paper.

1.1. Automorphic representations for Hilbert modular groups. Let $F$ be a totally real number field with degree $d$ over $\mathbb{Q}$. The Lie group $G=\mathrm{SL}_{2}(\mathbb{R})^{d}$ is the product $\prod_{j} \mathrm{SL}_{2}\left(k_{j}\right)$ over all infinite places $j$ of $F$. We fix a non-zero ideal $I$ in the ring of integers $\mathcal{O}$ of $F$. The group $G$ contains the discrete subgroup

$$
\Gamma_{0}(I)=\left\{\left(\begin{array}{ll}
a & b \\
c & d
\end{array}\right) \in \mathrm{SL}_{2}(\mathcal{O}): c \in I\right\}
$$

with finite covolume. 
Let $\chi$ be a character of $(\mathcal{O} / I)^{*}$. It determines a character of $\Gamma_{0}(I)$ by $\chi\left(\begin{array}{ll}a & b \\ c & d\end{array}\right)=$ $\chi(d)$. Let $L^{2}\left(\Gamma_{0}(I) \backslash G, \chi\right)$ be the Hilbert space of classes of functions transforming according to $f(\gamma g)=\chi(\gamma) f(g)$ for $\gamma \in \Gamma_{0}(I)$. The group $G$ acts unitarily in this Hilbert space by right translation. This space is split up according to central characters, indicated by $\xi \in\{0,1\}^{d}$. By $L_{\xi}^{2}\left(\Gamma_{0}(I) \backslash G, \chi\right)$ we mean the subspace on which the center acts by

$$
\left(\left(\begin{array}{cc}
\zeta_{1} & 0 \\
0 & \zeta_{1}
\end{array}\right), \ldots,\left(\begin{array}{cc}
\zeta_{d} & 0 \\
0 & \zeta_{d}
\end{array}\right)\right) \mapsto \prod_{j} \zeta_{j}^{\xi_{j}},
$$

where $\zeta_{j} \in\{1,-1\}$. This subspace can be non-zero only if the following compatibility condition holds:

$$
\chi(-1)=\prod_{j}(-1)^{\xi_{j}} .
$$

We assume this throughout the paper.

There is an orthogonal decomposition

$$
L_{\xi}^{2}\left(\Gamma_{0}(I) \backslash G, \chi\right)=L_{\xi}^{2, \text { cont }}\left(\Gamma_{0}(I) \backslash G, \chi\right) \oplus L_{\xi}^{2, \operatorname{discr}}\left(\Gamma_{0}(I) \backslash G, \chi\right) .
$$

The $G$-invariant subspace $L_{\xi}^{2, \text { cont }}\left(\Gamma_{0}(I) \backslash G, \chi\right)$ can be described by integrals of Eisenstein series, and the orthogonal complement $L_{\xi}^{2 \text {,discr }}\left(\Gamma_{0}(I) \backslash G, \chi\right)$ is a direct sum of closed irreducible $G$-invariant subspaces. If $\chi=1$, the constant functions form an invariant subspace. All other irreducible invariant subspaces have infinite dimension. They are cuspidal and span the space $L_{\xi}^{2, \operatorname{cusp}}\left(\Gamma_{0}(I) \backslash G, \chi\right)$, the orthogonal complement of the constant functions in $L_{\xi}^{2, \operatorname{discr}}\left(\Gamma_{0}(I) \backslash G, \chi\right)$.

We fix a maximal orthogonal system $\left\{V_{\varpi}\right\}_{\varpi}$ of irreducible subspaces in the Hilbert space $L_{\xi}^{2, \text { cusp }}\left(\Gamma_{0}(I) \backslash G, \chi\right)$. This system is unique if all $\varpi$ are inequivalent. In general, there might be multiplicities, due to oldforms.

Each irreducible automorphic representation $\varpi$ of $G=\prod_{j} \mathrm{SL}_{2}(\mathbb{R})$ is the tensor product $\bigotimes_{j} \varpi_{j}$ of irreducible representations of $\mathrm{SL}_{2}(\mathbb{R})$. Here and in the sequel, $j$ is supposed to run over the $d$ archimedean places of $F$.

The factor $\varpi_{j}$ can (almost) be characterized by the eigenvalue $\lambda_{\varpi, j}$ of the Casimir operator of $\mathrm{SL}_{2}(\mathbb{R})$ and by the central character, which is indicated by $\xi_{j}$.

If $\xi_{j}=0$, then the eigenvalue $\lambda_{\varpi, j}$ can either be of the form $\frac{b}{2}-\frac{b^{2}}{4}$ with $b \geq 2$ even or else the $\lambda_{\varpi, j}$ have a lower bound $\lambda_{0} \in\left(0, \frac{1}{4}\right]$. By the Selberg conjecture, it is expected that one can take $\lambda_{0}=\frac{1}{4}$. The best result at present is $\frac{77}{324}=\frac{1}{4}-\left(\frac{1}{9}\right)^{2} \leq$ $\lambda_{0} \leq \frac{1}{4}$; see [13. If $\xi_{j}=1$, then the $\lambda_{\varpi, j}$ can either lie in $\left[\frac{1}{4}, \infty\right)$ or be of the form $\frac{b}{2}-\frac{b^{2}}{4}$ with $b \geq 3, b$ odd. We call $\lambda_{\varpi}=\left(\lambda_{\varpi, j}\right) \in \mathbb{R}^{d}$ the eigenvalue vector of the representation $\varpi$.

Spectral theory shows that the set $\left\{\lambda_{\varpi}\right\}$ is discrete in $\mathbb{R}^{d}$. To see this we use the fact that the Casimir operator of $G$ has a discrete spectrum with finite multiplicities in $L_{\xi}^{2, \operatorname{cusp}}(\Gamma \backslash G)_{q}$, where $L_{\xi}^{2 \text {,cusp }}(\Gamma \backslash G)_{q}$ is the subspace of $L_{\xi}^{2 \text {,cusp }}(\Gamma \backslash G)$ with $K$-type (or weight) $q \in \mathbb{Z}^{d}, q \equiv \xi \bmod 2$. Hence the number of representations $\varpi$ (with multiplicities) such that $V_{\varpi} \cap L_{\xi}^{2 \text {,cusp }}(\Gamma \backslash G)_{q} \neq\{0\}$ and for which the eigenvalue $\lambda_{\varpi, 1}+\lambda_{\varpi, 2}+\cdots+\lambda_{\varpi, d}$ of the Casimir operator is under a given bound, is finite. For a given component $\varpi_{j}$ in the discrete series, the weights $q_{j}$ occurring in $V_{\varpi, j}$ satisfy $\left|q_{j}\right| \geq b_{j} \geq 1$, with $\lambda_{\varpi, j}=\frac{b_{j}}{2}\left(1-\frac{b_{j}}{2}\right)$. So for a given bounded set $\Omega \subset \mathbb{R}^{d}$ we 
can choose the $K$-type $q$ such that all $\varpi$ with $\lambda_{\varpi} \in \Omega$ are present in $L_{\xi}^{2, \operatorname{cusp}}(\Gamma \backslash G)_{q}$. Thus $\Omega$ contains only finitely many $\lambda_{\varpi}$, counted with multiplicities.

The correspondence between values of $\lambda=\lambda_{\varpi, j}$ and equivalence classes of unitary representations of $\mathrm{SL}_{2}(\mathbb{R})$ of infinite dimension is one-to-one if $\lambda>0$ for $\xi=\xi_{j}=0$, and if $\lambda>\frac{1}{4}$ if $\xi=1$. In the other cases, $\lambda=\frac{b}{2}-\frac{b^{2}}{4}$ with $b \in \mathbb{Z}_{\geq 1}$, $b \equiv \xi \bmod 2$. In these cases, there are two equivalence classes, one with lowest weight $b$ (holomorphic type) and one with highest weight $-b$ (antiholomorphic type). If $b \geq 2$, representations of these classes occur discretely in $L^{2}\left(\mathrm{SL}_{2}(\mathbb{R})\right)$ and are called discrete series representations. The representations in the case $b=1$ are sometimes called mock discrete series. They do not occur discretely in $L^{2}\left(\mathrm{SL}_{2}(\mathbb{R})\right)$.

All these classes of representations, discrete series or not, may occur as an irreducible component of $L_{\xi}^{2, \text { cusp }}\left(\Gamma_{0}(I) \backslash G, \chi\right)$.

As discussed in $\S 2.3 .4$ in [3], the Fourier expansion of one automorphic form in $V_{\varpi}$ determines the Fourier expansion of any automorphic form in $V_{\varpi}$. We refer to [3] for the normalization. This results in coefficients $c^{r}(\varpi)$ describing the Fourier expansion at the cusp $\infty$. The Fourier term order $r$ runs through the inverse different $\mathcal{O}^{\prime}$.

In the choice of the $c^{r}(\varpi)$ there is a freedom of a complex factor with absolute value one for a given $\varpi$. Since we shall work with weights $\left|c^{r}(\varpi)\right|^{2}$, this freedom has no influence on the results to which we aim.

When dealing with the sum formula, it is technically easier to parametrize the eigenvalues $\lambda_{\varpi, j} \in \mathbb{R}$ by $\lambda_{\varpi, j}=\frac{1}{4}-\nu_{\varpi, j}^{2}$, with, for instance, $\nu_{\varpi, j} \in(0, \infty) \cup i[0, \infty)$. We put $\nu_{\varpi}=\left(\nu_{\varpi, j}\right)$, and we call $\nu_{\varpi}$ and $\xi_{\varpi}=\left(\xi_{\varpi, j}\right) \in\{0,1\}^{d}$ the spectral parameters of $\varpi$.

We have $\nu_{\varpi} \in Y_{\xi}=\prod_{j} Y_{\xi_{j}}$, with

$$
\begin{aligned}
& Y_{0}:=\left\{\frac{b-1}{2}: b \geq 2 \text { even }\right\} \cup i[0, \infty) \cup\left(0, \nu_{0}\right], \\
& Y_{1}:=\left\{\frac{b-1}{2}: b \geq 3 \text { odd }\right\} \cup i[0, \infty),
\end{aligned}
$$

where $\nu_{0}=\sqrt{\frac{1}{4}-\lambda_{0}}$.

\subsection{Discussion of main results.}

1.2.1. Counting function. For compact sets $\Omega \subset \mathbb{R}^{d}$, we use the counting functions

$$
\mathrm{N}^{r}(\Omega):=\mathrm{N}_{\xi, \chi}^{r}(\Omega):=\sum_{\varpi, \lambda_{\varpi} \in \Omega}\left|c^{r}(\varpi)\right|^{2}
$$

with $r \in \mathcal{O}^{\prime} \backslash\{0\}$. The representations $\varpi$ run through the orthogonal system of irreducible subspaces of $L_{\xi}^{2, \operatorname{cusp}}\left(\Gamma_{0}(I) \backslash G, \chi\right)$ chosen in 1.1 . If there are multiplicities larger than one, the choice of the orthogonal system does not influence the counting. It may happen that $c^{r}(\varpi)=0$ for some $\varpi$ and some $r$. However, varying $r$, we can detect all $\varpi$.

More generally, if $f$ is a function on $\mathbb{R}^{d}$, we can consider the sum

$$
\mathrm{N}^{r}(f):=\mathrm{N}_{\xi, \chi}^{r}(f):=\sum_{\varpi} f\left(\lambda_{\varpi}\right)\left|c^{r}(\varpi)\right|^{2},
$$

which converges for suitable $f$, for instance, compactly supported $f$. So $\mathrm{N}^{r}(\Omega)=$ $\mathrm{N}^{r}\left(\chi_{\Omega}\right)$, where $\chi_{\Omega}$ is the characteristic function of $\Omega$. 
In the $\nu$-coordinate the counting function is

$$
\tilde{\mathrm{N}}^{r}(\tilde{\Omega})=\sum_{\varpi, \nu_{\varpi} \in \tilde{\Omega}}\left|c^{r}(\varpi)\right|^{2}
$$

for sets $\tilde{\Omega} \subset Y_{\xi}$.

1.2.2. Plancherel measure. We will compare $\mathrm{N}^{r}(\Omega)$ with $\mathrm{Pl}(\Omega)=\operatorname{Pl}_{\xi}(\Omega)$. The measure $\mathrm{Pl}$ on $\mathbb{R}^{d}$ is the product $\mathrm{Pl}=\bigotimes_{j} \mathrm{Pl}_{\xi_{j}}$, where $\mathrm{Pl}_{\xi_{j}}$ is the measure on $\mathbb{R}$ given by

$$
\begin{aligned}
\mathrm{Pl}_{0}(f)= & \int_{1 / 4}^{\infty} f(\lambda) \tanh \pi \sqrt{\lambda-\frac{1}{4}} d \lambda \\
& +\sum_{b \geq 2, b \equiv 0 \bmod 2}(b-1) f\left(\frac{b}{2}\left(1-\frac{b}{2}\right)\right), \\
\mathrm{Pl}_{1}(f)= & \int_{1 / 4}^{\infty} f(\lambda) \operatorname{coth} \pi \sqrt{\lambda-\frac{1}{4}} d \lambda \\
& +\sum_{b \geq 3, b \equiv 1 \bmod 2}(b-1) f\left(\frac{b}{2}\left(1-\frac{b}{2}\right)\right) .
\end{aligned}
$$

Note that $\mathrm{Pl}_{\xi_{j}}$ gives zero measure to the set of exceptional eigenvalues in $\left[\lambda_{0}, \frac{1}{4}\right)$.

The notation $\mathrm{Pl}$ refers to the Plancherel measure of $\mathrm{SL}_{2}(\mathbb{R})$; see, e.g., [15], Chap. VIII, §4, p. 174.

In the $\nu$-coordinate the Plancherel measure on $Y_{\xi}$ is given by $\widetilde{\mathrm{P}} l_{\xi}=\bigotimes_{j} \widetilde{\mathrm{Pl}}_{\xi_{j}}$, where

$$
\widetilde{\mathrm{Pl}}_{\xi_{j}}(f)=2 \int_{0}^{\infty} f(i t) \widetilde{\mathrm{pl}}_{j}(t) d t+2 \sum_{\beta \in \frac{\xi_{j}+1}{2}+\mathbb{N}_{0}} f(\beta) \widetilde{\mathrm{pl}}_{j}(\beta)
$$

with

\begin{tabular}{|c|c|c|}
\hline & & $\widetilde{\mathrm{pl}}_{j}(t)$ \\
\hline$\xi_{j}=0$ & $t \in i \mathbb{R}$ & $|t| \tanh \pi|t|$ \\
\hline$\xi_{j}=1$ & $t \in i \mathbb{R}$ & $|t| \operatorname{coth} \pi|t|$ \\
\hline$t \equiv \frac{\xi_{j}-1}{2} \bmod 1$ & $t \in \mathbb{R} \backslash\{0\}$ & $|t|$ \\
\hline$t \not \equiv \frac{\xi_{j}-1}{2} \bmod 1$ & $t \in \mathbb{R} \backslash\{0\}$ & 0 \\
\hline
\end{tabular}

If $f$ is even, then

$$
\begin{aligned}
& \widetilde{\mathrm{P}}_{0}(f)=i \int_{\operatorname{Re} \nu=0} f(\nu) \nu \tan \pi \nu d \nu+\sum_{\beta \in \frac{1}{2}+\mathbb{Z}}|\beta| f(\beta), \\
& \widetilde{\mathrm{P}}_{1}(f)=-i \int_{\operatorname{Re} \nu=0} f(\nu) \nu \cot \pi \nu d \nu+\sum_{\beta \in \mathbb{Z} \backslash\{0\}}|\beta| f(\beta) .
\end{aligned}
$$

The reference measure $V_{1}$ is more easily given in the $\nu$-coordinate. We leave the reformulation in terms of the $\lambda$-coordinate to the reader. The measure has a 
product form $\tilde{V}_{1}=\bigotimes_{j} \tilde{V}_{1, \xi_{j}}$ on the space $((0, \infty) \cup i[0, \infty))^{d}$ with

$$
\begin{aligned}
& \int h d \tilde{V}_{1,0}=\int_{1}^{\infty} t h(i t) d t+\int_{0}^{1} h(i t) d t+\int_{0}^{\nu_{0}} h(x) d x+\sum_{\beta>0, \beta \equiv \frac{1}{2}(1)} \beta h(\beta), \\
& \int h d \tilde{V}_{1,1}=\int_{1}^{\infty} t h(i t) d t+\int_{0}^{1} h(i t) d t+\sum_{\beta>0, \beta \equiv 0(1)} \beta h(\beta) .
\end{aligned}
$$

So $\tilde{V}_{1}$ is positive everywhere on $Y_{\xi}$, and $\widetilde{\mathrm{Pl}}(\tilde{\Omega}) \ll \tilde{V}_{1}(\tilde{\Omega})$ for all $\Omega$. We also have $\tilde{V}_{1}(\tilde{\Omega}) \ll_{\varepsilon} \widetilde{\mathrm{Pl}}(\tilde{\Omega})$ if the coordinates of $\tilde{\Omega}_{t}$ stay away from $\left(0, \nu_{0}\right] \cup i[0, \varepsilon)$ for some $\varepsilon>0$.

1.2.3. Asymptotic formula. We will prove that for families $t \mapsto \Omega_{t}$ of sets in $\mathbb{R}^{d}$, satisfying the conditions discussed below,

$$
\mathrm{N}^{r}\left(\Omega_{t}\right)=\frac{2 \sqrt{\left|D_{F}\right|}}{(2 \pi)^{d}} \operatorname{Pl}\left(\Omega_{t}\right)+o\left(V_{1}\left(\Omega_{t}\right)\right) \quad(t \rightarrow \infty)
$$

for any $r \in \mathcal{O}^{\prime} \backslash\{0\}$.

Here $D_{F}$ is the discriminant over $\mathbb{Q}$ of the totally real number field $F$ of degree $d$. The character $\chi$ of $(\mathcal{O} / I)^{*}$ and $\xi \in\{0,1\}^{d}$ are as explained in $\$ 1.1$.

The sets $\Omega_{t}$ should get large; in particular, $V_{1}\left(\Omega_{t}\right)$ should tend to infinity as $t \rightarrow \infty$. Moreover, the boundary $\partial \Omega_{t}$ should be relatively small in comparison with $\Omega_{t}$ itself. The precise conditions are discussed in 4.2 . Theorem 4.6 . Proposition 4.7 and Theorem 5.3 .

Since $\operatorname{Pl}\left(\Omega_{t}\right) \ll V_{1}\left(\Omega_{t}\right)$ the term $o\left(V_{1}\left(\Omega_{t}\right)\right)$ is small in comparison with $\operatorname{Pl}\left(\Omega_{t}\right)$. If $V_{1}\left(\Omega_{t}\right) \ll \operatorname{Pl}\left(\Omega_{t}\right)$ holds as well, then the asymptotic formula simplifies to

$$
\mathrm{N}^{r}\left(\Omega_{t}\right) \sim \frac{2 \sqrt{\left|D_{F}\right|}}{(2 \pi)^{d}} \operatorname{Pl}\left(\Omega_{t}\right) \quad(t \rightarrow \infty) .
$$

In this section we shall be content to discuss a number of families for which the asymptotic formula (19) holds, showing the existence of automorphic forms with eigenvalue (or spectral) parameters lying in such regions $\Omega_{t}$, as $t$ gets large.

1.2.4. Small rectangle in the real quadratic case. Before stating more general results, we consider the case that $d=2$, and we first apply some of the more general statements for this situation.

Let $[\alpha, \beta] \subset[1 / 4, \infty)$ and consider for $t \geq \frac{5}{4}$ :

$$
\Omega_{t}=[\alpha, \beta] \times[t, t+\sqrt{t}] .
$$

Theorem 1.3 implies

$$
\mathrm{N}^{r}\left(\Omega_{t}\right) \sim \frac{\sqrt{D_{F}}}{2 \pi^{2}} \int_{\alpha}^{\beta} \tanh \pi \sqrt{\lambda-\frac{1}{4}} d \lambda t^{1 / 2} \quad(t \rightarrow \infty) .
$$

In particular, there are infinitely many $\varpi$ with $\lambda_{\varpi, 1} \in[\alpha, \beta]$. These $\varpi$ have a second component of unitary principal series type. A similar result holds with $\varpi_{2}$ of discrete series type:

$$
\begin{aligned}
\Omega_{b} & =[\alpha, \beta] \times\left\{\frac{b-1}{2}\right\} \quad \text { with } b>1, b \cong \xi_{2} \bmod 2, \\
\mathrm{~N}^{r}\left(\Omega_{b}\right) & \sim \frac{\sqrt{D_{F}}}{2 \pi^{2}} \int_{\alpha}^{\beta} \tanh \pi \sqrt{\lambda-\frac{1}{4}} d \lambda \cdot b \quad(b \rightarrow \infty) .
\end{aligned}
$$


On the other hand if $[\alpha, \beta] \subset\left[\lambda_{0}, \frac{1}{4}\right)$, then

$$
\begin{aligned}
\text { for } \Omega_{t} & =[\alpha, \beta] \times[t, \sqrt{t}] \quad \text { with } t \geq \frac{5}{4}, \\
\mathrm{~N}^{r}\left(\Omega_{t}\right) & =o\left(t^{1 / 2}\right) \quad(t \rightarrow \infty) ; \\
\text { and for } \Omega_{b} & =[\alpha, \beta] \times\left\{\frac{b-1}{2}\right\} \quad \text { with } b>1, b \cong \xi_{2} \bmod 2, \\
\mathrm{~N}^{r}\left(\Omega_{b}\right) & =o(b) \quad(b \rightarrow \infty) .
\end{aligned}
$$

This does not exclude the presence of $\varpi_{1}$ of complementary series type, but gives an upper bound for their weighted density.

These results also hold with the role of $\varpi_{1}$ and $\varpi_{2}$ interchanged.

One may also consider families of rectangles for which both factors vary:

$$
\Omega_{t}=[t, t+\sqrt{t}] \times[c t, c t+\sqrt{t}],
$$

with $t \geq \frac{5}{4}$ and $c \geq 1$. Then

$$
\mathrm{N}^{r}\left(\Omega_{t}\right) \sim \frac{\sqrt{D_{F}}}{2 \pi^{2}} c^{1 / 2} t \quad(t \rightarrow \infty) .
$$

1.2.5. Floating boxes. We consider for general $F$ a small hypercube of fixed size floating to infinity in the region $(i[1, \infty))^{d}$ of the $\nu$-plane.

Proposition 1.1. Let $\tilde{\Omega}_{t}=\prod_{j} i\left[a_{j}(t), a_{j}(t)+\sigma\right]$ with $\sigma>0, a_{j}(t) \geq 1$ for all $j$ and $t$, and where $\lim _{t \rightarrow \infty} a_{j}(t)=\infty$ for at least one $j$. Then

$$
\tilde{\mathrm{N}}^{r}\left(\tilde{\Omega}_{t}\right) \sim \frac{2 \sqrt{\left|D_{F}\right|}}{(2 \pi)^{d}} \widetilde{\mathrm{Pl}}\left(\tilde{\Omega}_{t}\right) \quad(t \rightarrow \infty) .
$$

Note that $\widetilde{\mathrm{Pl}}\left(\tilde{\Omega}_{t}\right) \rightarrow \infty$. We have $\widetilde{\mathrm{Pl}}\left(\tilde{\Omega}_{t}\right) \sim \sigma^{d} \prod_{j} a_{j}(t)$ if $a_{j}(t) \rightarrow \infty$ for all $j$. Proposition 6.1 implies that the size $\sigma$ may even slowly decrease with $t$, provided

$$
\sigma(t) \geq \gamma\left(\sum_{j} \log a_{j}(t)\right)^{-\alpha} \quad \text { for any } \alpha \in\left(0, \frac{1}{2}\right), \gamma>0 .
$$

We conclude that there are spectral parameters $\nu_{\varpi}$ in such a hypercube if they are sufficiently far away from the origin. If we reformulate in terms of $\lambda$ we get boxes $\Omega_{t}$ in $\lambda$-space with increasing size.

1.2.6. Remark. On the other hand, for hypercubes in $\lambda$-space with fixed size, our method does not give an asymptotic formula. In fact, the $\lambda_{\varpi}$ may leave space for a small hypercube moving around in $\lambda$-space, avoiding all of the $\lambda_{\varpi}$. This can occur if the $\left|c^{r}(\varpi)\right|^{2}$ are not often very small.

1.2.7. Discrete series. For $\varpi$ for which all factors are discrete series representations, we do not need boxes, but we can work with single points.

Proposition 1.2. Let $\mathbf{p} \in \prod_{j}\left(\frac{\xi_{j}+1}{2}+\mathbb{N}_{0}\right)$ and let $\Omega=\Omega_{\mathbf{p}}=\{\mathbf{p}\}$. Then, if at least one coordinate of $\mathbf{p}$ tends to infinity, we have

$$
\tilde{\mathrm{N}}^{r}(\{\mathbf{p}\}) \sim \frac{2 \sqrt{\left|D_{F}\right|}}{\pi^{d}} \prod_{j} \mathbf{p}_{j}
$$


1.2.8. Combinations. A combination is possible. At some places we take a fixed interval $\left[A_{j}, B_{j}\right]$ on the $\lambda$-line. We have to require that the endpoints are not equal to a discrete series eigenvalue:

$$
A_{j}, B_{j} \notin\left\{\frac{b}{2}\left(1-\frac{b}{2}\right): b>1, b \equiv \xi_{j} \bmod 2\right\} .
$$

Theorem 1.3. Let $E \sqcup Q_{+} \sqcup Q_{-}$be a partition of the infinite places of $F$. Suppose that $B_{E}=\prod_{j \in E}\left[A_{j}, B_{j}\right]$ satisfies condition (28). Let

$$
C_{t}=\prod_{j \in Q_{+}} i\left[a_{j}(t), a_{j}(t)+\sigma\right]
$$

with $a_{j}(t) \geq 1$ for any $j \in Q_{+}$and all $t$, with $\delta>0$ fixed. Let $\mathbf{p} \in \prod_{j \in Q_{-}}\left(\frac{\xi_{j}+1}{2}+\mathbb{N}_{0}\right)$. Then, provided that $a_{j}(t) \rightarrow \infty$ for at least one $j \in Q_{+}$or $\mathbf{p}_{j} \rightarrow \infty$ for at least one $j \in Q_{-}$, we have

$$
\begin{array}{r}
\tilde{\mathrm{N}}^{r}\left(\tilde{B}_{E} \times C_{t} \times\{\mathbf{p}\}\right)=\frac{2 \sqrt{\left|D_{F}\right|}}{(2 \pi)^{d}} \widetilde{\mathrm{Pl}}\left(\tilde{B}_{E} \times C_{t} \times\{\mathbf{p}\}\right) \\
+o\left(\tilde{V}_{1}\left(\tilde{B}_{E} \times C_{t} \times\{\mathbf{p}\}\right)\right) .
\end{array}
$$

In Theorem 1.3 some factors of $B_{E}$ may be in the region $\left[\lambda_{0}, \frac{1}{4}\right)$, giving

$$
\widetilde{\mathrm{Pl}}\left(\tilde{B}_{E} \times C_{t} \times\{\mathbf{p}\}\right)=0 .
$$

So here we need $\tilde{V}_{1}$ in the asymptotic formula.

Again, the constant size $\sigma$ in Theorem 1.3 can be replaced by

$$
\sigma(t)=\gamma\left(\sum_{j \in Q_{+}} \log a_{j}(t)+\sum_{j \in Q_{-}} \log \left(2 \mathbf{p}_{j}\right)\right)^{-\alpha} \quad\left(0<\alpha<\frac{1}{2}, \gamma>0\right) .
$$

1.2.9. Floating spheres. The following is analogous to an unweighted distribution result, based on the Selberg trace formula, given by Huntley and Tepper [10]:

Proposition 1.4. Let $B(\mathbf{m}, r)$ be a sphere in $\mathbb{R}^{d}$ with center $\mathbf{m}$ and radius $r$. Let $\mathbf{m}_{j} \geq r+1$ for all $j$, and suppose that $\mathbf{m}_{j}$ tends to infinity for all $j$ (not necessarily with the same speed). Then

$$
\tilde{\mathrm{N}}^{r}(i B(\mathbf{m}, r)) \sim 4 \sqrt{\left|D_{F}\right|} v_{d}\left(\frac{r}{\pi}\right)^{d} \prod_{j} \mathbf{m}_{j},
$$

where $v_{d}$ denotes the volume of the unit sphere in $\mathbb{R}^{d}$.

This asymptotic formula holds even if $r=r(\mathbf{m})$ is allowed to go down to zero no faster than $\left(\log \prod_{j \in Q_{+}} \mathbf{m}_{j}\right)^{-\alpha}$, with $\alpha<\frac{1}{2}$.

1.2.10. Slanted strips in the quadratic case.

Proposition 1.5. Let $d=2$, and put

$$
\tilde{\Omega}_{t}=\left\{\left(\nu_{1}, \nu_{2}\right) \in(i[1, \infty))^{2}: t \leq\left|\nu_{1}\right| \leq 2 t, a\left|\nu_{1}\right|+b \leq\left|\nu_{2}\right| \leq a\left|\nu_{1}\right|+c\right\},
$$

with $a>0, c>b$ fixed and targe. Then

$$
\tilde{\mathrm{N}}^{r}\left(\tilde{\Omega}_{t}\right) \sim \frac{14}{3 \pi^{2}} \sqrt{\left|D_{F}\right|} a(c-b) t^{3} \quad(t \rightarrow \infty) .
$$


Proposition 1.5 shows that we see infinitely many points $\nu_{\varpi}$ in a slanted direction. This slanted strip becomes a sector in $\lambda$-space. It opens up with a speed of the order $\left|\lambda_{1}\right|^{1 / 2}$.

1.2.11. Density. Theorem 1.3 directly implies the density results in Propositions 3.7 and 3.8 in [2, without the restriction $\chi=1$ and $\xi=0$. The idea is that we take $|E|=d-1$ in Theorem 1.3 and use $B_{E}$ to restrict $\left(\lambda_{\varpi, j}\right)_{j \in E}$ to a tiny set with positive Plancherel measure. The remaining coordinate of $\lambda_{\varpi}$ is allowed to range through a set in $\mathbb{R}$ with increasing Plancherel measure. This shows that there are infinitely many $\varpi$ with $\left(\lambda_{\varpi, j}\right)_{j \in E} \in B_{E}$.

If one of the factors $\left[A_{j}, B_{j}\right]$ of $B_{E}$ is contained in the interval $\left[\lambda_{0}, \frac{1}{4}\right)$ of exceptional eigenvalues, the Plancherel measure of $B_{E} \times C_{t}$ is zero. The asymptotic formula cannot give the absence of $\varpi$ with $\lambda_{\varpi, j} \in\left[A_{j}, B_{j}\right]$, but only that the density is small in comparison with $\tilde{V}_{1}\left(B_{E} \times C_{t}\right)$. We note that we project here along a coordinate axis in the natural product structure of $\mathbb{R}^{d}$ as the product of the archimedean completions of $F$.

1.2.12. Weighted Weyl laws. In this subsection we list some consequences of the asymptotic formula in the $\lambda$-parameter. We have that the asymptotic formula holds for $\Omega_{t}=[-t, t]^{d}$. This implies

\section{Proposition 1.6.}

$$
\sum_{\varpi,\left|\lambda_{\varpi, j}\right| \leq t \text { for all } j}\left|c^{r}(\varpi)\right|^{2} \sim \frac{2 \sqrt{\left|D_{F}\right|}}{\pi^{d}} t^{d} \quad(t \rightarrow \infty) .
$$

This result confirms that there are infinitely many cuspidal $\varpi$ for each choice of $\chi$ and $\xi$ satisfying (8). With the normalization of the $c^{r}(\varpi)$ that we have chosen, the density does not depend on the order of the Fourier coefficients that we use.

Proposition 1.7. Let $Q_{+} \sqcup Q_{-}$be a partition of the real places of $F$. Then

$$
\begin{aligned}
& \sum_{\substack{\varpi \sum_{j}\left|\lambda_{\varpi, j}\right| \leq t \\
\lambda_{\varpi, j} \leq 0, j \in Q_{-} \\
\lambda_{\varpi, j}>0, j \in Q_{+}}}\left|c^{r}(\varpi)\right|^{2} \sim \frac{2 \sqrt{\left|D_{F}\right|}}{d !(2 \pi)^{d}} t^{d} .
\end{aligned}
$$

This variant is Corollary 3.4 in [2. There we considered only the trivial character of $\Gamma_{0}(I)$ and the trivial central character. Proposition 6.3 implies that all results in [2] extend to the more general context in this paper.

\subsubsection{Sectors in the quadratic case.}

Proposition 1.8. Let $d=2$, and fix $0<p<q, \alpha>\frac{1}{2}$. For $t \geq \frac{5}{4}\left(1+p^{-1}\right)$ put

$$
\Omega_{t}=\left\{\left(\lambda_{1}, \lambda_{2}\right) \in[0, \infty)^{2}: t \leq \lambda_{1} \leq t+t^{\alpha}, p \lambda_{1} \leq \lambda_{2} \leq q \lambda_{1}\right\} .
$$

Then

$$
\mathrm{N}^{r}\left(\tilde{\Omega}_{t}\right) \sim \frac{q-p}{4} t^{1+\alpha} \quad(t \rightarrow \infty)
$$




\section{Sum Formula}

The basis of the result in this paper is the Kuznetsov type sum formula in Theorem 3.21 of [3], which we recall in 2.1. In 2.2 we apply it with test functions adapted to the present purpose.

2.1. Statement of the sum formula. The sum formula as stated in [3] depends on two non-zero Fourier term orders $r, r^{\prime} \in \mathcal{O}^{\prime} \backslash\{0\}$. For the end results of this paper it suffices to take $r^{\prime}=r$. In a later paper we intend to work with different Fourier term orders and take Hecke operators into account. Then we'll need to consider $r \neq r^{\prime}$ as well.

The sum formula states an equality with four terms, all depending on a given test function. We first state the sum formula and will next recall the description of the ingredients.

Theorem 2.1 (Spectral sum formula.). For any test function $\varphi \in T_{\xi}(\tau, a)$ the sums and integrals $\tilde{\mathrm{N}}^{r}(\varphi)$, $\operatorname{Eis}^{r}(\varphi), \widetilde{\mathrm{Pl}}(\varphi)$ and $\mathrm{K}_{\chi}^{r}\left(\mathrm{~B}_{\xi}^{\mathbf{s}} \varphi\right)$ converge absolutely, and

$$
\tilde{\mathrm{N}}^{r}(\varphi)+\operatorname{Eis}^{r}(\varphi)=\frac{2 \sqrt{\left|D_{F}\right|}}{(2 \pi)^{d}} \widetilde{\mathrm{Pl}}(\varphi)+\mathrm{K}_{\chi}^{r}\left(\mathrm{~B}_{\xi}^{\mathbf{s}} \varphi\right) .
$$

We work with a fixed character $\chi$ of $\Gamma$ and a compatible central character given by $\xi \in\{0,1\}^{d}$; so condition (8) is satisfied. Fixed is $r \in \mathcal{O}^{\prime} \backslash\{0\}$. Also fixed are the parameters $\tau \in\left(\frac{1}{4}, \frac{1}{2}\right)$ and $a>2$, which determine the space of test functions.

2.1.1. Test functions. The space $T_{\xi}(\tau, a)$ of test functions consists of functions with the following product structure:

$$
\varphi\left(\nu_{1}, \ldots, \nu_{d}\right)=\prod_{j} \varphi_{j}\left(\nu_{j}\right),
$$

where each $\varphi_{j}$ is a function on a set

$$
\{z \in \mathbb{C}:|\operatorname{Re} z| \leq \tau\} \cup\left\{\frac{b-1}{2}: b \equiv \xi_{j} \bmod 2, b \geq 2\right\}
$$

satisfying the following conditions:

(T1) $\varphi_{j}$ is holomorphic on $|\operatorname{Re} z| \leq \tau$,

(T2) $\varphi_{j}(z) \ll(1+|z|)^{-a}$ on the domain of $\varphi_{j}$,

(T3) $\varphi_{j}(-z)=\varphi_{j}(z)$ on the strip $|\operatorname{Re} z| \leq \tau$.

2.1.2. Spectral side. On the left hand side of (32) are two terms connected to the spectral decomposition of $L_{\xi}^{2}\left(\Gamma_{0}(I) \backslash G, \chi\right)$. The first term $\tilde{\mathrm{N}}^{r}(\varphi)$ is the sum defined in (13). Its convergence already implies the existence of infinitely many cuspidal automorphic representations in $L_{\xi}^{2, \operatorname{cusp}}\left(\Gamma_{0}(I) \backslash G, \chi\right)$.

The orthogonal complement in $L_{\xi}^{2}\left(\Gamma_{0}(I) \backslash G, \chi\right)$ of the cuspidal subspace gives rise to the term $\operatorname{Eis}^{r}(\varphi)$. Since $r$ is non-zero, the constant functions, in the case $\chi=1, \xi=0$, do not contribute to the sum formula. We have

$$
\operatorname{Eis}^{r}(\varphi)=\sum_{\kappa \in \mathcal{P}_{\chi}} c_{\kappa} \sum_{\mu \in \Lambda_{\kappa, \chi}} \int_{-\infty}^{\infty}\left|D_{\xi}^{r}(\kappa, \chi ; i t, i \mu)\right| \varphi(i t+i \mu) d t
$$

Here $\mathcal{P}_{\chi}$ is a set of representatives of cuspidal orbits suitable for the character $\chi$. For each $\kappa$, there is a lattice $\Lambda_{\kappa, \chi}$ in the hyperspace $\sum_{j} x_{j}=0$ in $\mathbb{R}^{d}$. In $i t+i \mu$, the real number $t$ is identified to $(t, t, \ldots, t) \in \mathbb{R}^{d}$. The positive constants $c_{\kappa}$ come 
from the spectral formula for the continuous spectrum. The $D_{\xi}^{r}(\kappa, \chi ; i t, i \mu)$ are normalized Fourier coefficients of Eisenstein series. See (2.31) of [3]. There is a positive real number $q$ such that

$$
D_{\xi}^{r}(\kappa, \chi ; i t, i \mu) \ll_{F, I, r} \begin{cases}(\log (2+|t|))^{q}+\left(\log \max _{j}\left(\left|\mu_{j}\right|+1\right)\right)^{q} & \text { if } \mu \neq 0, \\ (\log (2+|t|))^{q} & \text { if } \mu=0 .\end{cases}
$$

This is discussed in Proposition 5.2 in 2 and $\S 4.2$ in 3 , with $q=7$.

Actually, it is conceivable that for some fields $F$, some ideals $I$ and some cusps $\kappa$, there might be $\mu \in \Lambda_{\kappa, \chi}$ with $\mu \neq 0$ and $\max _{j}\left|\mu_{j}\right|<1$. This is not intended in 2. The logarithms arise in (71) and (72) of 2]. Checking the reasoning there, we see that if the bounds for $Z(s, \lambda, \tau)$ are negative, they can be replaced by 0 . Thus, we can replace $\left(\log \max _{j}\left(\left|\mu_{j}\right|+1\right)\right)^{7}$ in (36) by $\left(\log \left(\max _{j}\left(\left|\mu_{j}\right|+2\right)\right)\right)^{7}$.

There is another reformulation. Put $x_{i}=t+\mu_{i}$. Then $t=\frac{1}{d} \sum_{j} x_{j}$ and $\mu_{i}=$ $x_{i}-\frac{1}{d} \sum_{j} x_{j}$. This implies that we have $|t| \ll \sum_{j}\left|x_{j}\right|$ and $\|\mu\| \ll \sum_{j}\left|x_{j}\right|$. Thus we arrive at

$$
D_{\xi}^{r}(\kappa, \chi ; i t, i \mu) \ll_{F, I, r}\left(\log \left(2+\sum_{j}\left|t+\mu_{j}\right|\right)\right)^{7} .
$$

2.1.3. Delta term. The right hand side of (32) arises from geometrical considerations. For the purpose of this paper the first term is the principal one.

2.1.4. Bessel transformation. The last term in (32) contains a Bessel transform of the test function. It depends on $r \in \mathcal{O}^{\prime} \backslash\{0\}$ by $\mathbf{s}=\left(\operatorname{sign} r_{j}\right)_{j} \in\{1,-1\}^{d}$. Here we view elements of $F$ as elements of $\mathbb{R}^{d}=\prod_{j} F_{j}$. The Bessel transformation has a product form:

$$
\begin{aligned}
\mathrm{B}_{\xi}^{\mathbf{s}} \varphi(t)= & \prod_{j} \mathrm{~B}_{\xi_{j}}^{\mathbf{s}_{j}} \varphi_{j}\left(t_{j}\right), \\
\mathrm{B}_{0}^{\eta} \varphi(t):= & -\frac{i}{2} \int_{\operatorname{Re} \nu=0} \varphi(\nu) \frac{J_{2 \nu}(|t|)-J_{-2 \nu}(|t|)}{\cos \pi \nu} \nu d \nu \\
& +\sum_{b \geq 2, b \equiv 0 \bmod 2}(-1)^{b / 2}(b-1) \varphi\left(\frac{b-1}{2}\right) J_{b-1}(|t|), \\
\mathrm{B}_{1}^{\eta} \varphi(t):= & -\frac{\eta}{2} \operatorname{sign}(t) \int_{\operatorname{Re} \nu=0} \varphi(\nu) \frac{J_{2 \nu}(|t|)+J_{-2 \nu}(|t|)}{\sin \pi \nu} \nu d \nu \\
& -i \eta \operatorname{sign}(t) \sum_{b \geq 3, b \equiv 1 \bmod 2}(-1)^{(b-1) / 2}(b-1) \varphi\left(\frac{b-1}{2}\right) J_{b-1}(|t|) .
\end{aligned}
$$

These Bessel transforms converge absolutely for any test function and provide us with functions $f=\mathrm{B}_{\xi}^{\mathbf{s}} \varphi$ on $\left(\mathbb{R}^{*}\right)^{d}$ that satisfy

$$
f(t) \ll \prod_{j} \min \left(\left|t_{j}\right|^{2 \tau}, 1\right) .
$$

2.1.5. Sum of Kloosterman sums. The Kloosterman sums for the present situation are

$$
S_{\chi}\left(r^{\prime}, r ; c\right)=\sum_{a \bmod (c)}^{*} \chi(a) e^{2 \pi i \operatorname{Tr}_{F / \mathbb{Q}}\left(\left(r a+r^{\prime} \tilde{a}\right) / c\right)},
$$


with $r, r^{\prime} \in \mathcal{O}^{\prime}$ and $c \in I \backslash\{0\}$. This is well defined if $\chi$ is a character of $(\mathcal{O} /(c))^{*}$, in particular for the character $\chi$ of $(\mathcal{O} / I)^{*}$ if $c \in I$. For each $a \in \mathcal{O}$ that is invertible modulo $(c)$, an element $\tilde{a}$ is picked such that $a \tilde{a} \equiv 1 \bmod (c)$.

For functions $f$ satisfying (39) the sum

$$
\mathrm{K}_{\chi}^{r}(f):=\sum_{c \in I \backslash\{0\}}|N(c)|^{-1} S_{\chi}(r, r ; c) f\left(\frac{4 \pi|r|}{c}\right)
$$

converges absolutely. This holds in particular for $f=\mathrm{B}_{\xi}^{\mathbf{S}} \varphi$. By $\frac{4 \pi|r|}{c}$ we mean the element of $\left(\mathbb{R}^{*}\right)^{d}$ given by the $d$ embeddings $F \rightarrow \mathbb{R}$ of $r$ and $c$.

A trivial estimate of the Kloosterman sums is $\left|S_{\chi}\left(r^{\prime}, r ; c\right)\right| \leq|N(c)|$. As discussed in $\S 2.4$ of [3], there is a Weil type estimate. If the ideal $(c)$ has a representation $(c)=\prod_{\mathfrak{p}} \mathfrak{p}^{v_{\mathfrak{p}}(c)}$ in prime ideals, then

$$
\left|S_{\chi}\left(r^{\prime}, r ; c\right)\right| \ll_{\delta}\left|N\left(r r^{\prime}\right)\right|^{1 / 2} \prod_{\mathfrak{p} \chi I}(N \mathfrak{p})^{v_{\mathfrak{p}}(c) / 2+\delta} \prod_{\mathfrak{p} \mid I}(N \mathfrak{p})^{v_{\mathfrak{p}}(c)+\delta}
$$

for each $\delta>0$. This is not the best possible estimate, however it is reasonably simple and will do for our purpose.

2.2. Application of the sum formula. In view of the term $\tilde{N}^{r}(\varphi)$ in the sum formula, we want to choose the test function $\varphi$ such that it approximates the characteristic function of a compact set $\tilde{\Omega}$ in the space $Y_{\xi}$ in which the spectral parameters $\nu_{\varpi}$ take their values. In this paper we first choose a test function approximating the delta distribution at $q \in \prod_{j \in Q} Y_{\xi_{j}}$ for a non-empty subset $Q$ of real places. At the other archimedean places we leave $\varphi_{j}$ free for the moment in the space of local test functions. The local factor $\varphi_{j}$ for $j \in Q$ such that $q_{j}=\frac{b-1}{2}$, $b \cong \xi_{j} \bmod 2, b \geq 2$, can be chosen such that practically $\varphi_{j}$ is the delta distribution at $\varphi_{j}$. For $q_{j} \in\left[0, \nu_{0}\right) \cup i[0, \infty)$ the choice is more delicate. It does not suffice that $\varphi_{j}$ approximates the delta distribution at $q_{j}$. The terms $\operatorname{Eis}^{r}(\varphi)$ and $\mathrm{K}^{r}\left(\mathrm{~B}_{\xi}^{\mathbf{s}} \varphi\right)$ should have good estimates. Under the additional assumption that $q_{j} \notin\left(0, \nu_{0}\right] \cup i[0,1]$, the choice that worked best is a sharp Gaussian function, similar to, but slightly simpler than, the test function used in, for instance, [11] and [12]. For $q_{j} \in\left(0, \nu_{0}\right] \cup i[0,1)$ we have not found a test function that works well.

Lemma 2.2. Let $\{1, \ldots, d\}=E \sqcup Q_{+} \sqcup Q_{-}$with $Q:=Q_{+} \cup Q_{-} \neq \emptyset$. For $q \in\left(\mathbb{R} \backslash\left(-\frac{1}{2}, \frac{1}{2}\right)\right)^{Q_{-}} \times(i \mathbb{R} \backslash i(-1,1))^{Q_{+}}$put $\varphi(q, \nu)=\prod_{j} \varphi\left(q_{j}, \nu_{j}\right)$, where $\varphi_{j}(q, \cdot)$ is an arbitrary local test function satisfying the conditions in 2.1 .1 if $j \in E$, and where for $j \in Q$,

\begin{tabular}{|l|l|}
\hline & $\varphi_{j}(q, \nu)$ \\
\hline$j \in Q_{+}$ & $\begin{cases}\sqrt{\frac{U}{\pi}}\left(e^{U(q-\nu)^{2}}+e^{U(q+\nu)^{2}}\right) & \text { if }|\operatorname{Re} \nu| \leq \tau \\
0 & \text { elsewhere }\end{cases}$ \\
\hline$j \in Q_{-}$ & $\begin{cases}1 & \text { if } \nu=q \text { or }-q \\
0 & \text { elsewhere }\end{cases}$ \\
\hline
\end{tabular}


Then there are constants $t_{0}>0, \rho \in(1-\tau, 1)$ such that for $U \geq 1$ and $A>2$,

$$
\begin{aligned}
& \tilde{\mathrm{N}}^{r}(\varphi(q, \cdot))=\frac{2 \sqrt{\left|D_{F}\right|}}{(2 \pi)^{d}} \widetilde{\mathrm{Pl}}(\varphi(q, \cdot)) \\
& \quad+O_{F, I, r, t_{0}, t_{1}, A}\left(N_{E}\left(\varphi_{E}\right) e^{t_{0} U\left|Q_{+}\right|} \prod_{j \in Q_{+}}\left|q_{j}\right|^{\rho} \prod_{j \in Q_{-}}\left|q_{j}\right|^{-A}\right),
\end{aligned}
$$

where $\varphi_{E}=\bigotimes_{j \in E} \varphi_{j}$ and

$$
\begin{aligned}
N_{E}\left(\varphi_{E}\right) & =\prod_{j \in E} N_{j}\left(\varphi_{j}\right), \\
N_{j}\left(\varphi_{j}\right) & =\sup _{\nu, 0 \leq \operatorname{Re} \nu \leq \tau}|\varphi(\nu)|(1+|\nu|)^{a}+\sum_{b \equiv \xi_{j}(2), b \geq 2} b^{a}\left|\varphi_{j}\left(\frac{b-2}{2}\right)\right| .
\end{aligned}
$$

Note that $\varphi_{j}$ chosen in (43) is a local test function for any choice of the parameters $a>2$ and $\tau \in\left(\frac{1}{4}, \frac{1}{2}\right)$.

By Theorem 2.1 it suffices to estimate $\operatorname{Eis}^{r}(\varphi(q, \cdot))$ and $\mathrm{K}_{\chi}^{r}\left(\mathrm{~B}_{\xi}^{\mathbf{S}} \varphi(q, \cdot)\right)$ by the error term in (44). This we carry out in the remainder of this subsection.

2.2.1. Bessel transforms. For the factors $j \in E$ we cannot do better than to apply Lemma 3.12 in [3]. This gives

$$
\mathrm{B}_{\xi_{j}}^{\mathbf{s}_{j}, \mathbf{s}_{j}^{\prime}} \varphi_{j}(t) \ll_{E} \min \left(|t|^{2 \tau}, 1\right) .
$$

The subscript $E$ in $\ll_{E}, O_{E}$ and $o_{E}$ indicates not only dependence on the choice of the set $E$, but also on the choice of the test function $\varphi_{E}:=\bigotimes_{j \in E} \varphi_{j}$. Here and in the sequel, this dependence goes via the factor $N_{E}\left(\varphi_{E}\right)$ in (45).

For $j \in Q_{-}$we first consider $y \leq 2 \sqrt{n}$, with $n \in \mathbb{N}$. Then $\left|J_{n}(y)\right| \leq \frac{(y / 2)^{n} e^{\frac{1}{4} y^{2}}}{n !} \ll$ $y^{2 \tau} \frac{n^{\frac{1}{2} n-\tau} e^{n}}{(n+1)^{n+\frac{1}{2}} e^{-n}} \ll y^{2 \tau}\left(\frac{n e^{4}}{(n+1)^{2}}\right)^{\frac{1}{2} n} n^{-\frac{1}{2}-\tau} \ll_{b} \frac{y^{2 \tau}}{n^{b}}$ for each $b>0$. For $y \geq \sqrt{n}$, $\left|J_{n}(y)\right| \leq 1$. Hence, for $j \in Q_{-}$and $\pm q_{j} \in \frac{\xi_{j}-1}{2}+\mathbb{N}$,

$$
\mathrm{B}_{\xi_{j}}^{\mathbf{s}_{j}} \varphi_{j}(q ; t) \ll_{A} \min \left(|q|^{-A_{1}}|t|^{2 \tau},|q|\right) \quad \text { for each } A_{1}>0 .
$$

If $\left|q_{j}\right| \notin \frac{\xi_{j}-1}{2}+\mathbb{N}$, then $\mathrm{B}_{\xi_{j}}^{\mathbf{s}_{j}, \mathbf{s}_{j}} \varphi_{j}(t)=0$.

The case $j \in Q_{+}$takes more work. The function $\varphi_{j}(q, \nu)$ is non-zero only for $|\operatorname{Re} \nu| \leq \tau$. We need an estimate like (47), in which the dependence on $q$ is explicit.

We may use (3.64) in [3]:

$$
\mathrm{B}_{\xi_{j}}^{\mathbf{s}_{j}} \varphi_{j}(q ; t)=-i\left(i \mathbf{s}_{j} \operatorname{sign} t\right)^{\xi_{j}} \int_{\operatorname{Re} \nu=\tau} \varphi_{j}(q, \nu) \frac{\nu J_{2 \nu}(|t|)}{\cos \pi\left(\nu-\frac{\xi_{j}}{2}\right)} d \nu .
$$

We proceed as in $\S 4.2$ of [2], and apply the integral representation in (41) of loc. cit., with $\alpha=\operatorname{Re} \nu=\tau$ and $\gamma \in\left(\tau, \frac{1}{2}\right)$. As in 2], p. 700, this leads to the estimate that for all $|t|>0$ :

$$
J_{2 \nu}(|t|) \ll|t|^{2 \tau} e^{\pi|\operatorname{Im} \nu|}(1+|\operatorname{Im} \nu|)^{\frac{1}{2}-\gamma-\tau} .
$$


We obtain for $q \in i \mathbb{R}$ :

$$
\begin{aligned}
& \mathrm{B}_{\xi_{j}}^{\mathbf{s}_{j}} \varphi_{j}(q ; t) \ll U^{1 / 2} \int_{-\infty}^{\infty} \sum_{ \pm} e^{U \operatorname{Re}(i x+\tau \pm q)^{2}}|t|^{2 \tau}|\tau+i x| \\
& \quad \cdot(1+|x|)^{\frac{1}{2}-\tau-\gamma} d x \\
& \ll|t|^{2 \tau} U^{1 / 2} \int_{-\infty}^{\infty} e^{U \tau^{2}-U(x-|q|)^{2}}(1+|x|)^{\frac{3}{2}-\gamma-\tau} d x \\
& \ll|t|^{2 \tau} e^{U \tau^{2}} \int_{0}^{\infty} e^{-x^{2}}\left(1+|q|+\frac{x}{\sqrt{U}}\right)^{\frac{3}{2}-\gamma-\tau} d x \\
& \ll|t|^{2 \tau} e^{U \tau^{2}} \int_{0}^{\infty} e^{-x^{2}}\left((1+|q|)^{\frac{3}{2}-\gamma-\tau}+\left(x U^{-1 / 2}\right)^{\frac{3}{2}-\gamma-\tau}\right) d x \\
& \ll|t|^{2 \tau} e^{U \tau^{2}}\left((1+|q|)^{\frac{3}{2}-\gamma-\tau}+U^{-\frac{3}{4}+\frac{\gamma}{2}+\frac{\tau}{2}}\right) \\
& \ll|t|^{2 \tau} e^{U \tau^{2}}(1+|q|)^{\rho_{1}}
\end{aligned}
$$

where $\rho_{1}=\frac{3}{2}-\gamma-\tau$; so $\rho_{1} \in\left(1-\tau, \frac{3}{2}-2 \tau\right) \subset\left(\frac{1}{2}, 1\right)$.

The factor $e^{U \tau^{2}}$ needs attention since it becomes large for large values of $U$. We carry along this factor and will compensate for it later.

These estimates are good for small values of $|t|$. For large $|t|$, we use

$$
J_{2 \nu}(|t|) \ll e^{\pi|\operatorname{Im} \nu|} \quad \text { for } \operatorname{Re} \nu=0 ;
$$

see (5.44) in [3], with $\sigma=0$.

$$
\begin{aligned}
\mathrm{B}_{\xi_{j}}^{\mathbf{s}_{j}} \varphi_{j}(q, t) & \ll U^{1 / 2} \int_{\operatorname{Re} \nu=0}\left|e^{U(\nu+q)^{2}}+e^{U(\nu-q)^{2}}\right||\nu||d \nu| \\
& \ll \int_{-\infty}^{\infty} e^{-x^{2}}\left|x U^{-1 / 2}+\right| q|| d x \ll 1+|q| \ll|q| .
\end{aligned}
$$

Thus we have

$$
\mathrm{B}_{\xi}^{\mathrm{s}} \varphi(q ; t) \ll_{A_{1}} \prod_{j}\left(a_{j}\left|t_{j}\right|^{2 \tau}, b_{j}\right),
$$

with

\begin{tabular}{|c|c|c|}
\hline$a_{j}$ & $b_{j}$ & \\
\hline$N_{j}\left(\varphi_{j}\right)$ & $N_{j}\left(\varphi_{j}\right)$ & $j \in E$ \\
\hline$|q|^{-A_{1}}$ & $|q|$ & $j \in Q_{-}$ \\
\hline$e^{\frac{1}{2} \tau^{2} U}|q|^{\rho_{1}}$ & $|q|$ & $j \in Q_{+}$ \\
\hline
\end{tabular}

2.2.2. Kloosterman term. We estimate the sum of Kloosterman sums by the sum of the absolute values of the terms:

$$
\mathrm{K}_{\chi}^{r, r}\left(\mathrm{~B}_{\xi}^{\mathbf{s}, \mathbf{s}} \varphi(q ; \cdot)\right) \ll \sum_{c \in I \backslash\{0\}} \frac{\left|S_{\chi}(r, r ; c)\right|}{|N(c)|} \prod_{j} \min \left(a_{j}\left(\frac{4 \pi\left|r_{j} r_{j}^{\prime}\right|^{1 / 2}}{\left|c_{j}\right|}\right)^{2 \tau}, b_{j}\right),
$$

with $a_{j}$ and $b_{j}$ as in (53). 
For the Kloosterman sums, we use the Weil bound, as stated in (42). This bound depends only on the ideal $(c)$, so we decompose the sum as

$$
\begin{aligned}
& \ll_{r, \delta} \sum_{(c) \subset I} \prod_{\mathfrak{p} \chi I} N \mathfrak{p}^{v_{\mathfrak{p}}(c)(\delta-1 / 2)} \prod_{\mathfrak{p} \mid I} N \mathfrak{p}^{v_{\mathfrak{p}}(c) \delta} \\
& \cdot \sum_{\zeta \in \mathcal{O}^{*}} \prod_{j} \min \left(p_{j}\left|\zeta_{j}\right|^{-2 \tau}, q_{j}\right), \\
& \text { with } \quad p_{j}=a_{j}\left(4 \pi\left|r_{j}\right|\right)^{2 \tau}, \quad q_{j}=b_{j}, \quad(c)=\prod_{\mathfrak{p} \text { prime }} \mathfrak{p}^{v_{\mathfrak{p}}(c)} .
\end{aligned}
$$

The prime denotes that the zero ideal is excluded. We can take $\delta>0$ as small as we want.

We apply Lemma 2.2 in [3] with $\alpha=2 \tau, \beta=0$ and $y_{j}=c_{j}^{-1}$. Thus, we estimate the sum over $\zeta \in \mathcal{O}^{*}$ by

$$
\begin{aligned}
& \ll\left(1+|\log | N(c)\left|+\frac{1}{2 \tau} \log \frac{\mathbf{Q}}{\mathbf{P}}\right|\right)^{d-1} \min \left(\mathbf{P}|N(c)|^{-2 \tau}, \mathbf{Q}\right), \\
\mathbf{P} & =\prod_{j} p_{j}=(4 \pi)^{2 \tau d}|N(r)|^{2 \tau} e^{\frac{1}{2} \tau^{2} U\left|Q_{+}\right|} N_{E}\left(\varphi_{E}\right) \prod_{j \in Q_{+}}\left|q_{j}\right|^{\rho_{1}} \prod_{j \in Q_{-}}\left|q_{j}\right|^{-A_{1}}, \\
\mathbf{Q} & =\prod_{j} q_{j}=N_{E}\left(\varphi_{E}\right) \prod_{j \in Q}\left|q_{j}\right|, \\
\frac{\mathbf{P}}{\mathbf{Q}} & =(4 \pi)^{2 \tau d} e^{\frac{1}{2} \tau^{2} U\left|Q_{+}\right|}|N(r)|^{2 \tau} \prod_{j \in Q_{+}}\left|q_{j}\right|^{\rho_{1}-1} \prod_{j \in Q_{-}}\left|q_{j}\right|^{-A_{1}-1} .
\end{aligned}
$$

We already have a small quantity $\delta$. We employ it also for the logarithms. We use the fact that for $c \in \mathcal{O} \backslash\{0\}$ the $|N(c)|$ stay away from zero:

$$
\begin{gathered}
|\log | N(c)\left|+\frac{1}{2 \tau} \log \frac{\mathbf{Q}}{\mathbf{P}}\right| \leq|\log | N(c)||+\frac{1}{2 \tau}\left|\log \frac{\mathbf{Q}}{\mathbf{P}}\right| \\
\ll_{\delta}|N(c)|^{\delta /(d-1)}+\max \left(\left(\frac{\mathbf{Q}}{\mathbf{P}}\right)^{\delta /(d-1)},\left(\frac{\mathbf{P}}{\mathbf{Q}}\right)^{\delta /(d-1)}\right), \\
|\log | N(c)\left|+\frac{1}{2 \tau} \log \frac{\mathbf{Q}}{\mathbf{P}}\right|^{d-1} \ll|N(c)|^{\delta}+\max \left(\left(\frac{\mathbf{Q}}{\mathbf{P}}\right)^{\delta},\left(\frac{\mathbf{P}}{\mathbf{Q}}\right)^{\delta}\right), \\
\left(1+|\log | N(c)\left|+\frac{1}{2 \tau} \log \frac{\mathbf{Q}}{\mathbf{P}}\right|\right) \min \left(\mathbf{P}|N(c)|^{-2 \tau}, \mathbf{Q}\right) \\
\ll_{r} e^{\frac{1}{2} \tau^{2} U\left|Q_{+}\right|(1+\delta)} N_{E}\left(\varphi_{E}\right)|N(c)|^{-2 \tau(1-\delta)} \\
\cdot \prod_{j \in Q_{+}}\left|q_{j}\right|^{\rho+\left(1-\rho_{1}\right) \delta} \prod_{j \in Q_{-}}\left|q_{j}\right|^{-A_{1}+\left(A_{1}-1\right) \delta} .
\end{gathered}
$$

We have assumed that the first factor in the minimum is the essential one for our purpose.

Let us define: $t_{0}=\frac{1}{2} \tau^{2}(1+\delta), \tau_{1}=\tau(1+\delta), \rho=\rho_{1}+\left(1-\rho_{1}\right) \delta$, and $A=$ $A_{1}+\left(1-A_{1}\right) \delta$. We take $A_{1}=-3$ and $\delta>0$ sufficiently small such that $\frac{1}{4}<\tau_{1}<\frac{1}{2}$, $1-\tau<\rho<1, A>2$. We find the following estimate for the sum of Kloosterman 
sums in (54):

$$
\begin{aligned}
& \ll_{r, \delta} e^{t_{0} U\left|Q_{+}\right|} N_{E}\left(\varphi_{E}\right) \sum_{(c) \subset I}^{\prime} \prod_{j \in Q_{+}}\left|q_{j}\right|^{\rho} \\
& \cdot \prod_{j \in Q_{-}}\left|q_{j}\right|^{-A} \prod_{\mathfrak{p} \backslash I} N \mathfrak{p}^{v_{\mathfrak{p}}(c)\left(\delta-1 / 2-2 \tau_{1}\right)} \prod_{\mathfrak{p} \mid I} N \mathfrak{p}^{v_{\mathfrak{p}}(c)\left(\delta-2 \tau_{1}\right)} \\
& \ll e^{t_{0} U\left|Q_{+}\right|} N_{E}\left(\varphi_{E}\right) \prod_{j \in Q_{+}}\left|q_{j}\right|^{\rho} \prod_{j \in Q_{-}}\left|q_{j}\right|^{-A} \\
& \cdot \prod_{\mathfrak{p} \backslash I} \frac{1}{1-N \mathfrak{p}^{\delta-1 / 2-2 \tau_{1}}} \prod_{\mathfrak{p} \mid I} \frac{1}{1-N \mathfrak{p}^{\delta-2 \tau_{1}}} .
\end{aligned}
$$

Under the additional assumption on $\delta$ that $2 \tau_{1}+\frac{1}{2}-\delta>1$, the product converges, and we have obtained

$$
\mathrm{K}_{\chi}^{r, r}\left(\mathrm{~B}_{\xi}^{\mathbf{s}, \mathbf{s}} \varphi(q ; \cdot)\right) \ll_{F, I, r, \delta} e^{t_{0} U\left|Q_{+}\right|} N_{E}\left(\varphi_{E}\right) \prod_{j \in Q_{+}}\left|q_{j}\right|^{\rho} \prod_{j \in Q_{-}}\left|q_{j}\right|^{-A},
$$

with the size of the error term in (44).

This is the main place where the dependence on the ideal $I \subset \mathcal{O}$ determining $\Gamma=\Gamma_{0}(I) \subset \mathrm{SL}_{2}(\mathcal{O})$ enters the estimates. We leave this dependence implicit.

2.2.3. Eisenstein term. We still have to estimate $\operatorname{Eis}^{r}(\varphi(q, \cdot))$. The definition in (35) Shows that $\operatorname{Eis}^{r}(\varphi(q, \cdot))=0$ if $Q_{-}=0$.

For $j \in E$, we have $\varphi_{j}(\nu) \ll_{E}\left(1+|\nu|^{2}\right)^{-a}$. In view of (37) it suffices to estimate

$$
\begin{gathered}
\sum_{\kappa \in \mathcal{P}_{\chi}} \sum_{\mu \in \Lambda_{\kappa, \chi}} \int_{-\infty}^{\infty}|N(r)|^{2 \delta} N_{E}\left(\varphi_{E}\right) l(q, t+\mu) d t, \text { with } \\
l(q, x)=\prod_{j \in E}\left(1+x_{j}^{2}\right)^{\delta-a / 2} \\
\cdot \prod_{j \in Q_{+}} U^{1 / 2}\left(1+x_{j}^{2}\right)^{\delta}\left(e^{-U\left(x_{j}-\left|q_{j}\right|\right)^{2}}+e^{-U\left(x_{j}+\left|q_{j}\right|\right)^{2}}\right) .
\end{gathered}
$$

In $\S 5.3$ of [2], we have replaced the sum over $\mu \in \Lambda_{\kappa, 1}$ by an integral over the hyperplane $\sum_{j=1}^{d} x_{j}=0$. Since in [2] the quantity corresponding to $U$ went down to zero, there was no problem there. Here $U$ may be large, and we have to take a closer look at the relation between the sum and the integral.

The integral gives a contribution, under the assumption $\frac{a}{2}-\delta>1$ :

$$
\begin{gathered}
\int_{\mathbb{R}^{d}} l(q, x) d x \ll \prod_{j \in E} \int_{-\infty}^{\infty}\left(1+x^{2}\right)^{\delta-a / 2} d x \\
\cdot \prod_{j \in Q_{+}} U^{1 / 2} \int_{-\infty}^{\infty}\left(1+x^{2}\right)^{\delta} e^{-U(x-|q|)^{2}} d x \\
\ll \prod_{j \in E} 1 \prod_{j \in Q_{+}} \int_{-\infty}^{\infty}\left(1+\left|q_{j}\right|^{2}+U^{-1} x^{2}\right)^{\delta} e^{-x^{2}} d x \\
\ll \prod_{j \in Q_{+}}\left(1+\left|q_{j}\right|\right)^{2 \delta}\left(1+\frac{1}{U}\right) \ll \prod_{j \in Q_{+}}\left|q_{j}\right|^{2 \delta} .
\end{gathered}
$$


The difference between the value at $\mu \in \Lambda_{\kappa, \chi}$ and the integral over $\mu+V$, where $V$ is a compact neighborhood of 0 , produces an error estimated by the gradient of $l(q, \cdot)$ :

$$
\frac{\partial}{\partial x_{j}} l(q, x) \ll_{a, \delta} l(q, x) \cdot \begin{cases}\frac{\left|x_{j}\right|}{1+x_{j}^{2}} & \text { if } j \in E, \\ \left(\frac{\left|x_{j}\right|}{1+x_{j}^{2}}+U\left|x_{j}-\right| q_{j}||\right) & \text { if } j \in Q_{+} .\end{cases}
$$

The difference between the sum and the integral is estimated by

$$
\int_{\mathbb{R}^{d}}\left(\sum_{m \in E} O(1)+\sum_{m \in Q_{+}}\left(1+\left.U\left|x_{m}-\right| q\right|_{m} \mid\right)\right) l(q, x) d x .
$$

The terms with $m \in E$ can be estimated as in (55). For a term with $m \in Q_{+}$,

$$
\begin{aligned}
& \ll \int_{-\infty}^{\infty}\left(1+\left.U\left|x_{m}-\right| q\right|_{m} \mid\right) U^{1 / 2}\left(1+x_{m}^{2}\right) e^{-U\left(x-\left|q_{m}\right|\right)^{2}} d x \\
& \quad \cdot \prod_{j \in E} O(1) \prod_{j \in Q_{+} \backslash\{m\}}\left|q_{j}\right|^{2 \delta} \\
& \ll \prod_{j \in Q_{+} \backslash\{m\}}\left|q_{j}\right|^{2 \delta} \\
& \quad \cdot \int_{-\infty}^{\infty}\left(1+U^{1 / 2}|x|\right)\left(1+\left|q_{m}\right|^{2}+U^{-1} x^{2}\right)^{\delta} e^{-x^{2}} d x \\
& \ll U^{1 / 2} \prod_{j \in Q_{+}}\left|q_{j}\right|^{2 \delta} .
\end{aligned}
$$

Thus, we obtain

$$
\operatorname{Eis}^{r}(\varphi(q, \cdot)) \ll_{F, I, r, \delta} N_{E}\left(\varphi_{E}\right) U^{1 / 2} \prod_{j \in Q_{+}}\left|q_{j}\right|^{2 \delta} .
$$

Since $\delta>0$ can be as small as we desire, this bound is easily absorbed into the error term in (44).

\subsubsection{Delta term.}

Lemma 2.3. Let $\widetilde{\mathrm{pl}}_{j}$ as in (16). For $E, Q_{+}, Q_{-}$and $\varphi(q, \cdot)$ as in Lemma 2.2 ,

$$
\begin{aligned}
\widetilde{\mathrm{Pl}}_{\xi}(\varphi(q, \cdot))= & \prod_{j \in E} \widetilde{\mathrm{P}}_{\xi_{j}}\left(\varphi_{j}\right) \prod_{j \in Q} 2 \widetilde{\mathrm{pl}}_{j}\left(q_{j}\right) \\
& + \begin{cases}U^{-1 / 2} N_{E}\left(\varphi_{E}\right)\left|Q_{+}\right| \frac{\prod_{j \in Q}\left|q_{j}\right|}{\min _{j \in Q_{+}}\left|q_{j}\right|} & \text { if } Q_{+} \neq \emptyset, \\
0 & \text { if } Q_{+}=\emptyset .\end{cases}
\end{aligned}
$$

Proof. Since

$$
\widetilde{\mathrm{Pl}}_{\xi_{j}}\left(\varphi_{j}(q, \cdot)\right)= \begin{cases}2|q| & \text { if } 2 q \equiv \xi_{j}-1 \bmod 2 \\ 0 & \text { otherwise }\end{cases}
$$

we have to consider the discrepancy between $\widetilde{\mathrm{Pl}}_{\xi_{j}}\left(\varphi_{j}(q, \cdot)\right)$ and $2 \widetilde{\mathrm{pl}}{ }_{j}\left(q_{j}\right)$ for $j \in Q_{+}$.

The function $t \mapsto \widetilde{\mathrm{pl}}_{j}(i t)$ is even and smooth on $\mathbb{R}$. If $\xi_{j}=0$, then $\widetilde{\mathrm{pl}}_{j}(0)=0$, and if $\xi_{j}=1$, then $\widetilde{\mathrm{pl}}_{j}(0)=\frac{1}{\pi}$. We have $\mathrm{pl}_{j}(i t) \sim|t|$ as $|t| \rightarrow \infty$, and $\frac{d}{d t} \mathrm{pl}_{j}(i t)=O(1)$ 
for $t \in \mathbb{R}$. Then

$$
\begin{aligned}
\widetilde{\mathrm{Pl}}_{\xi_{j}} & \left(\varphi_{j}\left(q_{j}, \cdot\right)\right)-2 \widetilde{\mathrm{pl}}_{j}\left(q_{j}\right) \\
& =2 \sqrt{\frac{U}{\pi}} \int_{0}^{\infty}\left(e^{-\left(x-\left|q_{j}\right|\right)^{2}}+e^{-(x+|q|)^{2}}\right) \widetilde{\mathrm{pl}}_{j}(i x) d x-2 \widetilde{\mathrm{pl}}_{j}\left(q_{j}\right) \\
& =2 \sqrt{\frac{U}{\pi}} \int_{-\infty}^{\infty} e^{-U\left(x-\left|q_{j}\right|\right)^{2}} \widetilde{\mathrm{pl}}_{j}(x) d x-2 \widetilde{\mathrm{pl}}_{j}\left(q_{j}\right) \\
& =\frac{2}{\sqrt{\pi}} \int_{-\infty}^{\infty} e^{-x^{2}}\left(\widetilde{\mathrm{pl}}_{j}\left(q_{j}+\frac{i x}{\sqrt{U}}\right)-\widetilde{\mathrm{pl}}_{j}\left(q_{j}\right)\right) d x
\end{aligned}
$$

We write $\widetilde{\mathrm{pl}}_{j}\left(q_{j}+\frac{i x}{\sqrt{U}}\right)-\widetilde{\mathrm{pl}}_{j}\left(q_{j}\right)=U^{-1 / 2} x \frac{d}{d \vartheta} \widetilde{\mathrm{pl}}_{j}(i \vartheta)$ for $|x| \leq b$, with $b \geq 1$, and $\vartheta$ between $\left|q_{j}\right|$ and $\left|q_{j}\right|+\frac{x}{\sqrt{U}}$. Then

$$
\begin{aligned}
\widetilde{\mathrm{Pl}}_{\xi_{j}} & \left(\varphi_{j}\left(q_{j}, \cdot\right)\right)-2 \widetilde{\mathrm{pl}}_{j}\left(q_{j}\right) \\
& \ll \int_{-b}^{b} e^{-x^{2}} O(1) U^{-1 / 2}|x| d x+\int_{|x| \geq b} e^{-x^{2}} O\left(\left|q_{j}\right|+|x| U^{-1 / 2}\right) d x \\
& \ll U^{-1 / 2}+\left|q_{j}\right| \frac{e^{-b^{2}}}{b}+U^{-1 / 2} e^{-b^{2}} .
\end{aligned}
$$

Here we have used the fact that for $b \in \mathbb{R}$ and $l \geq 0$,

$$
\int_{b}^{\infty}|x|^{l} e^{-x^{2}} d x \ll_{l} \begin{cases}1 & \text { if } b \leq 1, \\ b^{l-1} e^{-b^{2}} & \text { if } b \geq 1,\end{cases}
$$

which can be checked by partial integration and induction.

We assume that $U \geq e^{2}$, and choose $b=b(q, U)=\sqrt{\log \left|q_{j}\right|+\frac{1}{2} \log U}$, which satisfies $b \geq 1$. This gives

$$
\widetilde{\mathrm{Pl}}_{\xi_{j}}\left(\varphi_{j}\left(q_{j}, \cdot\right)\right)-2 \widetilde{\mathrm{pl}}_{j}\left(-i q_{j}\right) \ll U^{-1 / 2} .
$$

Furthermore, we have

$$
\begin{aligned}
\widetilde{\mathrm{pl}}_{j}\left(q_{j}\right) & \ll\left|q_{j}\right| & & \left(j \in Q_{+}\right), \\
\widetilde{\mathrm{Pl}}_{\xi_{j}}\left(\varphi_{j}\left(q_{j}, \cdot\right)\right) & \ll U^{-1 / 2}+\left|q_{j}\right| \ll\left|q_{j}\right| & & \left(j \in Q_{+}\right), \\
\widetilde{\mathrm{pl}}_{\xi_{j}}\left(\varphi_{j}\left(q_{j}, \cdot\right)\right) & \ll\left|q_{j}\right| & & \left(j \in Q_{-}\right), \\
\widetilde{\mathrm{Pl}}_{\xi_{j}}\left(\varphi_{j}\right) & \ll N_{j}\left(\varphi_{j}\right) & & (j \in E) .
\end{aligned}
$$

These local estimates imply that

$$
\begin{aligned}
\prod_{j \in Q_{+}} \widetilde{\mathrm{Pl}}_{\xi_{j}}\left(\varphi_{j}\left(q_{j}, \cdot\right)\right)-\prod_{j \in Q_{+}} 2 \tilde{\mathrm{pl}}_{j}\left(q_{j}\right) \ll \sum_{m \in Q_{+}} U^{-1 / 2} \prod_{j \in Q_{+}, j \neq m}\left|q_{j}\right| \\
\ll U^{-1 / 2}\left|Q_{+}\right| \frac{\prod_{j \in Q_{+}}\left|q_{j}\right|}{\min _{j \in Q_{+}}\left|q_{j}\right|} .
\end{aligned}
$$

Hence we have shown the estimate in (57). 
We now fix $A>2$. From here on we view the quantities $t_{0}>0$ and $\rho \in(1-\tau, 1)$ also as absolute quantities, like $\tau$ and $a$ in the sum formula. We apply Lemmas 2.2 and 2.3 to obtain:

Proposition 2.4. For $E, Q_{+}, Q_{-}, \varphi(q, \cdot)$ as in Lemma 2.2, with $U \geq e^{2}$,

$$
\begin{aligned}
\tilde{\mathrm{N}}^{r}(\varphi(q, \cdot)) & =\frac{2^{|Q|+1} \sqrt{\left|D_{F}\right|}}{(2 \pi)^{d}} \widetilde{\mathrm{Pl}}_{E}\left(\varphi_{E}\right) \prod_{j \in Q} \widetilde{\mathrm{pl}}_{j}\left(q_{j}\right) \\
& +O_{F, I, r}\left(N_{E}\left(\varphi_{E}\right) e^{t_{0} U\left|Q_{+}\right|} \prod_{j \in Q_{+}}\left|q_{j}\right|^{\rho} \prod_{j \in Q_{-}}\left|q_{j}\right|^{-A}\right) \\
& +O\left(N_{E}\left(\varphi_{E}\right) U^{-1 / 2}\left|Q_{+}\right| \frac{\prod_{j \in Q}\left|q_{j}\right|}{\min _{j \in Q_{+}}\left|q_{j}\right|}\right) .
\end{aligned}
$$

This is the basis for the results in the next sections.

The main term in (61) can be estimated by $O_{F}\left(N_{E}\left(\varphi_{E}\right) \prod_{j \in Q}\left|q_{j}\right|\right)$. This implies

$$
\tilde{\mathrm{N}}^{r}(\varphi(q, \cdot)) \ll_{F, I, r, U} N_{E}\left(\varphi_{E}\right) \prod_{j \in Q}\left|q_{j}\right|
$$

\section{UPPER BOUND}

The next step is to derive by integration of (62) an upper bound for $\tilde{\mathrm{N}}^{r}(f)$ for functions of the form $f=\varphi_{E} \otimes A:=\varphi_{E} \otimes \chi_{A}$, where $\chi_{A}$ is the characteristic function of a set $A$. To integrate, we fix a non-negative measure $d_{Q}$ on $((0, \infty) \cup i[0, \infty))^{Q}$ of the form $d_{Q} q=\bigotimes_{j \in Q} d_{j} q_{j}$ :

$$
\int h(q) d_{j} q=\int_{0}^{\infty} h(i t) d t+\int_{0}^{\nu_{0}} h(x) d x+\sum_{\beta>0, \beta \equiv \frac{\xi_{j}-1}{2}(1)} h(\beta) .
$$

We shall use $d_{R} q=\bigotimes_{j \in R} d_{j} q_{j}$ for any set $R$ of real places.

We define for $b \in \mathbb{R}$ and for bounded measurable sets $B \subset(\mathbb{R} \cup i \mathbb{R})^{R}$ with $R \subset Q$ :

$$
\begin{aligned}
\tilde{V}_{b}(B) & =\int_{B} \prod_{j \in R} p\left(q_{j}\right)^{b} d_{R} q, \\
p\left(q_{j}\right) & = \begin{cases}1 & \text { if } q_{j} \in\left(0, \nu_{0}\right] \cup i[0,1), \\
\left|q_{j}\right| & \text { otherwise. }\end{cases}
\end{aligned}
$$

The set $R$ of real places is not visible in the notation $\tilde{V}_{b}$ and should be clear from the set $B$. Note that with $b=1$ this definition agrees with (18).

For our purpose it suffices to estimate $\tilde{\mathrm{N}}^{r}(\varphi \otimes A)$ for bounded sets $A$ of the form

$$
\begin{aligned}
A & =A_{+} \times A_{0} \times A_{-}, & & A_{+} \subset(i[1, \infty))^{R_{+}}, \\
A_{0} & =\left(\left(0, \nu_{0}\right) \cup i[0,1)\right)^{R_{0}}, & & A^{-} \subset \prod_{j \in R_{-}}\left(\frac{\xi_{j}+1}{2}+\mathbb{N}_{0}\right),
\end{aligned}
$$

for any partition $Q=R_{+} \sqcup R_{0} \sqcup R_{-}$. This choice reflects that $q$ in (62) has no factors in $\left(0, \nu_{0}\right) \cup i[0,1)$. We have failed to find a test function that allows the sum formula to see sharply in this region. 
The aim is to estimate $\tilde{\mathrm{N}}^{r}\left(\varphi_{E} \otimes A\right)$ by $N_{E}\left(\varphi_{E}\right) \tilde{V}_{1}(A)$. Given perfect knowledge of the spectral set $\left\{\nu_{\varpi}\right\}$, one can choose $A_{+}$as the union of tiny boxes around many $\nu_{\varpi, A_{+}}=\left(\nu_{\varpi, j}\right)_{j \in A_{+}}$in such a way that $\mathrm{N}^{r}\left(\varphi_{E} \otimes A\right)$ is large while $\tilde{V}_{1}(A)$ stays arbitrarily small. This shows that we need a further assumption on the factor $A_{+}$.

Let dist be the distance along $i[0, \infty) \cup\left(0, \nu_{0}\right)$ given by

$$
\operatorname{dist}(\nu, q)= \begin{cases}|q-\nu| & \text { if } q, \nu \in i[0, \infty) \text { or } q, \nu \in\left(0, \nu_{0}\right) \\ |q|+|\nu| & \text { otherwise. }\end{cases}
$$

For $\varepsilon>0$ and $\nu \in\left(i[0, \infty) \cup\left(0, \nu_{0}\right)\right)^{B}$, where $B$ is a set of real places,

$$
A(\nu, \varepsilon)=\left\{q \in\left(i[0, \infty) \cup\left(0, \nu_{0}\right)\right)^{B}: \operatorname{dist}\left(q_{j}, \nu_{j}\right) \leq \frac{\varepsilon}{2} \text { for any } j \in B\right\} .
$$

Again, the set $B$ should be clear from the context.

Definition 3.1. Let $w, \varepsilon>0$, and let $B$ be a set of real places. A $(w, \varepsilon)$-blunt subset $H \subset\left(i[0, \infty) \cup\left(0, \nu_{0}\right]\right)^{B}$ is a $d_{B} q$-measurable set such that

$$
\int_{A\left(\nu_{R}, \beta\right) \cap H} d_{B} q \geq w \operatorname{vol}_{B} A(\nu, \beta) \quad \text { for all } \nu \in H \text { and all } \beta \in(0, \varepsilon] .
$$

By $\operatorname{vol}_{B}$ we mean the volume for $d_{B}$.

Note that $(w, \varepsilon)$-bluntness implies $\left(w, \varepsilon_{1}\right)$-bluntness for any $\varepsilon_{1} \in(0, \varepsilon)$. Boxes with size at least $\varepsilon$ in all coordinate directions are $(1, \varepsilon)$-blunt.

Proposition 3.2. Let $A=A_{+} \times A_{0} \times A_{-}$be as in (66). Suppose that $A_{+}$is $(w, \varepsilon)$-blunt for some $w>0$ and $\varepsilon \in\left(0, e^{-1}\right]$. Then for any $\varphi_{E}$,

$$
\mathrm{N}^{r}\left(\varphi_{E} \otimes A\right) \ll_{F, I, r} w^{-1} N_{E}\left(\varphi_{E}\right) \tilde{V}_{1}(A) .
$$

Proof. We apply Proposition 2.4 with $E$ replaced by $\hat{E}=E \cup R_{0}, Q_{ \pm}$replaced by $R_{ \pm}$, and the test function $\hat{\varphi}(q, \cdot)$ chosen as follows:

\begin{tabular}{|c|c|c|c|}
\hline$j \in$ & $E$ & $R_{+} \cup R_{-}$ & $R_{0}$ \\
\hline$\hat{\varphi}_{j}=$ & $\varphi_{p}$ & $\varphi_{j}(q, \cdot)$ with $U \geq e^{2}$ & $\begin{cases}e^{\nu^{2}} & \text { if }|\operatorname{Re} \nu| \leq \tau, \\
0 & \text { otherwise; }\end{cases}$ \\
\hline
\end{tabular}

$$
\varphi_{p}(\nu)= \begin{cases}\left(p^{2}-\nu^{2}\right)^{-a / 2} & \text { if }|\operatorname{Re} \nu| \leq \tau \\ \left(p^{2}+\nu^{2}\right)^{-a / 2} & \text { otherwise }\end{cases}
$$

with some fixed $p>\tau$ and $q \in(i[1, \infty))^{R_{+}} \times \prod_{j \in R_{-}}\left(\frac{\xi_{j}+1}{2}+\mathbb{N}_{0}\right)$. We put

$$
\varphi_{p, E}(\nu)=\bigotimes_{j \in E} \varphi_{p}\left(\nu_{j}\right)
$$

From (62) we obtain

$$
\tilde{\mathrm{N}}^{r}(\hat{\varphi}(q, \cdot)) \ll_{F, I, r} N_{E}\left(\varphi_{p, E}\right) \prod_{j \in R_{+} \cup R_{-}}\left|q_{j}\right|,
$$


where we have used the fact that (45) implies $N_{j}\left(\varphi_{j}\right)=O(1)$ for $j \in R_{0}$. Integration over $q$ gives

$$
\int_{A_{+} \times A_{-}} \tilde{\mathrm{N}}^{r}(\hat{\varphi}(q, \cdot)) d_{R_{+} \cup R_{-}} q \ll_{F, I, r} \tilde{V}_{1}\left(A_{+} \times A_{-}\right) .
$$

We have omitted $N_{E}\left(\varphi_{p, E}\right)$ since it is $O(1)$ for the fixed choice of $p$.

Now we note that for a given $\nu \in Y_{\xi}$ we have $\hat{\varphi}(q, \nu) \geq 0$. With the obvious meaning $\left(\nu_{j}\right)_{j \in B}$ of $\nu_{B}$ for sets $B$ of real places, we have

$$
\begin{aligned}
\int_{A_{+} \times A_{-}} & \hat{\varphi}(q, \nu) d_{R_{+} \cup R_{-}}=\int_{A_{+} \times\left\{\nu_{R_{-}}\right\}} \hat{\varphi}\left(q, \nu_{\varpi}\right) d_{R_{+} \cup R_{-}} q \\
& \geq \int_{A\left(\nu_{R_{+}}, \varepsilon\right) \cap A_{+}} \varphi_{p, E}\left(\nu_{E}\right) \prod_{j \in R_{0}} e^{\nu_{j}} \prod_{j \in R_{-}} 1 \prod_{j \in R_{+}} \varphi_{j}\left(q_{j}, \nu_{j}\right) d_{R_{+}} q \\
& \geq \varphi_{p, E}\left(\nu_{E}\right) e^{-\left|R_{0}\right|}\left(\sqrt{\frac{U}{\pi}} e^{-U \varepsilon^{2}}\right)^{\left|R_{+}\right|} \int_{A\left(\nu_{R_{+}}, \varepsilon\right) \cap A_{+}} d_{R_{+}} q \\
& \geq w \varphi_{p, E}\left(\nu_{E}\right) e^{-\left|R_{0}\right|}\left(\frac{\varepsilon U^{1 / 2} e^{-U \varepsilon^{2}}}{\pi^{1 / 2}}\right)^{\left|R_{+}\right|} .
\end{aligned}
$$

With the choice $U=\varepsilon^{-2}$,

$$
\int_{A_{+} \times A_{-}} \hat{\varphi}(q, \nu) d_{R_{+} \cup R_{-}} \geq w e^{-\left|R_{0}\right|} \pi^{-\left|R_{+}\right| / 2} \varphi_{p, E}\left(\nu_{E}\right) .
$$
in

Since $\hat{\varphi}(q, \cdot) \geq 0$ on $Y_{\xi}$, we can reverse the order of summation and integration

$$
\int_{A_{+} \times A_{-}} \tilde{\mathrm{N}}^{r}(\hat{\varphi}(q, \cdot)) d_{R_{+} \cup R_{-}} q=\sum_{\varpi}\left|c^{r}(\varpi)\right|^{2} \int_{A_{+} \times A_{-}} \hat{\varphi}\left(q, \nu_{\varpi}\right) d_{R_{+} \cup R_{-}} q .
$$

Hence

$$
\begin{aligned}
\tilde{\mathrm{N}}^{r}\left(\varphi_{E}\right. & \otimes A)=\sum_{\varpi, \nu_{\varpi, Q} \in A}\left|c^{r}(\varpi)\right|^{2} \varphi_{p, E}\left(\nu_{\varpi, E}\right) \\
& \leq \frac{e^{\left|R_{0}\right|}}{w} \pi^{\left|R_{+}\right| / 2} \sum_{\varpi, \nu_{\varpi} \in A}\left|c^{r}(\varpi)\right|^{2} \int_{A_{+} \times A_{-}} \hat{\varphi}(q, \nu) d_{R_{+} \cup R_{-}} q \\
& \ll_{d} \frac{1}{w} \int_{A_{+} \times A_{-}} \tilde{\mathrm{N}}^{r}(\hat{\varphi}(q, \cdot)) d_{R_{+} \cup R_{-}} q .
\end{aligned}
$$

With (73),

$$
\tilde{\mathrm{N}}^{r}\left(\varphi_{E} \otimes A\right) \ll_{F, I, r} \frac{1}{w} \tilde{V}_{1}(A),
$$

where we have used the fact that $\tilde{V}_{1}\left(A_{0}\right)=O(1)$.

\section{Asymptotic formula, first stage}

Now we start a more precise approximation of $\mathrm{N}^{r}\left(\varphi_{E} \times C\right)$, where $\varphi_{E}$ is still an arbitrary test function and where $C$ is a product $C^{+} \times C^{-}$, with bounded closed sets $C^{+} \subset(i[1, \infty))^{Q_{+}}$and $C^{-} \subset\left[\frac{1}{2}, \infty\right)^{Q_{-}}$. Only the intersection $C^{-} \cap$ 
$\prod_{j \in Q_{-}}\left(\frac{\xi_{j}+1}{2}+\mathbb{N}_{0}\right)$ matters for the present purpose, not $C^{-}$itself. For $C^{+}$we define, with $c>0$,

$$
\begin{aligned}
C^{+}(0) & =C^{+}, \\
C^{+}(c) & =\left\{\nu \in\left(i[0, \infty) \cup\left(0, \nu_{0}\right]\right)^{Q_{-}}: A(\nu, c) \cap C^{+} \neq \emptyset\right\}, \\
C^{+}(-c) & =\left\{\nu \in C^{+}: A(\nu, c) \subset C^{+}\right\}, \\
C^{+}[c] & =C^{+}(c) \backslash C^{+}(-c) .
\end{aligned}
$$

Proposition 4.1. Let $r \in \mathcal{O}^{\prime} \backslash\{0\}$. Let $a>2$ and $\tau \in\left[\frac{1}{4}, \frac{1}{2}\right]$ as in Theorem 2.1, and decompose the set of real places of $F$ as $E \sqcup Q_{+} \sqcup Q_{-}$with $Q=Q_{+} \cup Q_{-} \neq \emptyset$. There are $t_{0}>1, D>0, \rho \in(1-\tau, 1)$ and $A>2$ such that for any $U>D e^{2}$ and any $\varepsilon \in\left[\left(\frac{D}{U}\right)^{1 / 2}, e^{-1}\right]$, for all products $\varphi_{E}=\bigotimes_{j \in E} \varphi_{j}$ of local test functions and for all bounded $d_{Q}$-measurable sets $C=C^{+} \times C^{-}, C^{+} \subset(i[1, \infty))^{Q_{+}}$and $C^{-} \subset\left[\frac{1}{2}, \infty\right)^{Q_{-}}$,

$$
\tilde{\mathrm{N}}^{r}\left(\varphi_{E} \times C\right)=\frac{2 \sqrt{\left|D_{F}\right|}}{(2 \pi)^{d}} \widetilde{\mathrm{P}}\left(\varphi_{E} \otimes C\right)+N_{E}\left(\varphi_{E}\right) O_{F, I, r}(E(C, U, \varepsilon)),
$$

where if $Q_{+}=\emptyset$,

$$
E(C, U, \varepsilon)=\tilde{V}_{-A}\left(C^{-}\right),
$$

and if $Q_{+} \neq \emptyset$,

$$
\begin{array}{r}
E(C, U, \varepsilon)=e^{t_{0} U\left|Q_{+}\right|} \tilde{V}_{\rho}\left(C^{+}\right) \tilde{V}_{-A}\left(C^{-}\right)+e^{-U \varepsilon^{2}} \tilde{V}_{1}(C) \\
+\tilde{V}\left(C^{+}[2 \varepsilon] \times C^{-}\right)+U^{-1 / 2} \tilde{V}_{1}(C) .
\end{array}
$$

At this point we can derive the statement in example (iv) in the Introduction. We denote by $S_{b}(\Gamma, \chi)$ the space of holomorphic cusp forms on the product $\mathfrak{H}^{d}$ of $d$ copies of the upper half plane for the group $\Gamma$ with character $\chi$ and weight $b \in \mathbb{N}^{d}$ satisfying $b_{j} \geq 1$ and $b_{j} \equiv \xi_{j} \bmod 2$ for all $j$.

Corollary 4.2. The space $S_{b}\left(\Gamma_{0}(I), \chi\right)$ is non-zero for all but finitely many weights in the set $\left\{b \in \mathbb{N}^{d}: b_{j} \geq 2, b_{j} \equiv \xi_{j} \bmod 2\right.$ for all $\left.j\right\}$.

Proof. We apply Proposition 4.1 with $E=Q_{+}=\emptyset$, and $C=C^{-}$equal to the singleton $C_{b}=\left\{\left(\frac{b_{j}-1}{2}\right)_{j}\right\}$. Then $\widetilde{\operatorname{Pl}}\left(C_{b}\right)=\prod_{j=1}^{d} \frac{b_{j}-1}{2}$ and $\tilde{V}_{-A}\left(C_{b}\right)=\prod_{j=1}^{d}\left(\frac{b_{j}-1}{2}\right)^{-A}$. We obtain (6) with the constant $C$ equal to $2 \sqrt{\left|D_{F}\right|}(2 \pi)^{-d}$.

If we take $r$ totally positive, the $\varpi$ entering $\tilde{\mathrm{N}}^{r}\left(C_{b}\right)$ form an orthogonal system of cuspidal representations for which each factor $\varpi_{j}$ is a discrete series representation with lowest weight $b_{j}$. (See (2.29) in [3.) Thus these $\varpi$ correspond to an orthogonal basis of $S_{b}\left(\Gamma_{0}(I), \chi\right)$. So $\tilde{\mathrm{N}}^{r}\left(C_{b}\right)$ can be non-zero only if $S_{b}\left(\Gamma_{0}(I), \chi\right) \neq\{0\}$.

4.1. Proof of Proposition 4.1. The proof of the proposition is rather long and will require some intermediate steps that we shall give in a series of lemmas.

4.1.1. Integration. We integrate (61) over $C$. Taking into account (15), we obtain

$$
\begin{aligned}
\int_{c} \tilde{\mathrm{N}}^{r}(\varphi(q, \cdot)) d_{Q} q-\frac{2 \sqrt{\left|D_{F}\right|}}{(2 \pi)^{d}} \widetilde{\mathrm{Pl}}_{E}\left(\varphi_{E}\right) \widetilde{\mathrm{Pl}}_{Q}(C) \\
\quad \ll_{F, I, r} N_{E}\left(\varphi_{E}\right) e^{t_{0} U\left|Q_{+}\right|} \tilde{V}_{\rho}\left(C^{+}\right) \tilde{V}_{-A}\left(C^{-}\right)+N_{E}\left(\varphi_{E}\right) U^{-1 / 2} V_{1}(C) .
\end{aligned}
$$


In this term we have left out the denominator $\min _{j \in Q_{+}}\left|q_{j}\right|$, since we have already a small factor $U^{-1 / 2}$.

To prove Proposition 4.1 we will estimate the difference

$$
\int_{c} \tilde{\mathrm{N}}^{r}(\varphi(q, \cdot)) d_{Q} q-\tilde{\mathrm{N}}^{r}\left(\varphi_{e} \otimes C\right) .
$$

4.1.2. Local comparison. Let $X_{j}=X_{j}^{+}=\frac{\xi_{j}+1}{2}+\mathbb{N}_{0}$ if $j \in Q_{-}$, and $X_{j}=\left(0, \nu_{0}\right] \cup$ $i[0, \infty), X_{j}^{+}=i[1, \infty)$ if $j \in Q_{+}$. We consider for $\nu \in X_{j}$ :

$$
\begin{aligned}
& I_{\alpha}^{j}(\nu)=\int_{q \in X_{j}, \operatorname{dist}(q, \nu) \leq \alpha} \varphi_{j}(q, \nu) d_{j} q, \\
& J_{\alpha}^{j}(\nu)=\int_{q \in X_{j}, \operatorname{dist}(q, \nu) \geq \alpha} \varphi_{j}(q, \nu) d_{j} q .
\end{aligned}
$$

Lemma 4.3. If $\alpha \geq U^{-1 / 2}$, then for $j \in Q_{+}, \nu \in X_{j}$,

$$
\begin{array}{lll}
\text { if } \nu \in i[1+\alpha, \infty): & I_{\alpha}^{j}(\nu)=1+O\left(e^{-U \alpha^{2}}\right), & J_{\alpha}^{j}(\nu)=O\left(e^{-U \alpha^{2}}\right), \\
\text { if } \nu \in i[0,1+\alpha): & I_{\alpha}^{j}(\nu)=O(1), & J_{\alpha}^{j}(\nu)=O\left(e^{-U \alpha^{2}}\right) .
\end{array}
$$

For $j \in Q_{+}, \nu \in X_{j}, U^{-1 / 2} \leq \varepsilon \leq e^{-1}$, and $\nu \in i[0,1-\varepsilon) \cup\left(0, \nu_{0}\right]$,

$$
I_{\varepsilon}^{j}(\nu)=0, \quad J_{\varepsilon}^{j}(\nu)=O\left(e^{-U \varepsilon^{2}}\right) .
$$

For $j \in Q_{-}, \nu \in X_{j}$,

$$
I_{\alpha}^{j}(\nu)=1, \quad J_{\alpha}^{j}(\nu)=0 .
$$

Proof. The results for $j \in Q_{-}$are immediate. We consider the case $j \in Q_{+}$. The best situation is $\nu \in i[1+\alpha, \infty)$. Then, with (59),

$$
\begin{aligned}
I_{\alpha}^{j}(\nu) & =\sqrt{\frac{U}{\pi}} \sum_{ \pm} \int_{|\nu|-\alpha}^{|\nu|+\alpha} e^{-U(x \mp|\nu|)^{2}} d x \\
& =\frac{1}{\sqrt{\pi}}\left(\int_{-\alpha \sqrt{U}}^{\alpha \sqrt{U}} e^{-x^{2}}+\int_{(2|\nu|-\alpha) \sqrt{U}}^{(2|\nu|+\alpha) \sqrt{U}} e^{-x^{2}} d x\right) \\
& =1+O\left(\frac{e^{-U \alpha^{2}}}{\alpha \sqrt{U}}\right)+O\left(\frac{e^{-U(2|\nu|-\alpha)^{2}}}{(2|\nu|-\alpha) \sqrt{U}}\right)=1+O\left(\frac{e^{-U \alpha^{2}}}{\alpha \sqrt{U}}\right), \\
J_{\alpha}^{j}(\nu) & =\left(\int_{1}^{|\nu|-\alpha}+\int_{|\nu|+\alpha}^{\infty}\right) \sqrt{\frac{U}{\pi}} \sum_{ \pm} e^{-U(x \mp|\nu|)^{2}} d x \\
& \ll \frac{e^{-U \alpha^{2}}}{\alpha \sqrt{U}}+\frac{e^{-U(2|\nu|-\alpha)^{2}}}{(2|\nu|-\alpha) \sqrt{U}} \ll \frac{e^{-U \alpha^{2}}}{\alpha \sqrt{U}} .
\end{aligned}
$$

We ignore the denominator $\alpha \sqrt{U} \geq 1$.

If $\nu \in i[0,1+\alpha]$, part of the integral for $I_{\alpha}^{j}(\nu)$ is omitted. This leads to the estimate $O(1)$. The quantity $J_{\alpha}^{j}(\nu)$ is at most as large as in the previous case.

We consider finally a small value $\alpha=\varepsilon \in\left[U^{-1 / 2}, e^{-1}\right]$. For $\nu \in i[0,1-\varepsilon] \cup\left(0, \nu_{0}\right]$, the integral $I_{\varepsilon}^{j}(\nu)$ vanishes. If $\nu \in i[0,1-\varepsilon)$ we have already obtained $J_{\varepsilon}^{j}(\nu)=$ 
$O\left(e^{-U \varepsilon^{2}}\right)$. For $\nu \in\left(0, \nu_{0}\right] \subset\left(0, \frac{1}{2}\right]$,

$$
\begin{aligned}
J_{\varepsilon}^{j}(\nu) & =\sqrt{\frac{U}{\pi}} \int_{1}^{\infty} e^{U\left(\nu^{2}-t^{2}\right)} \cos 2 t \nu d t \\
& \ll e^{U \nu^{2}} \int_{\sqrt{U}}^{\infty} e^{-x^{2}} d x \ll \frac{e^{-U\left(1-\nu^{2}\right)}}{\sqrt{U}} \leq \frac{e^{-U\left(1-\nu_{0}^{2}\right)}}{1} \leq e^{-U \varepsilon^{2}} .
\end{aligned}
$$

\subsubsection{Global comparison.}

Lemma 4.4. Let $\nu \in Y_{\xi}, \nu_{j} \in X_{j}$ for $j \in Q$. Let $\alpha \geq U^{-1 / 2}$ and $\varepsilon \in\left[U^{-1 / 2}, e^{-1}\right]$. Then

$$
\begin{gathered}
\int_{q \in A\left(\nu_{Q_{+}}, \alpha\right) \times\left\{\nu_{Q_{-}}\right\}} \varphi(q, \nu) d_{Q} q \ll \varphi_{E}\left(\nu_{E}\right) ; \\
\int_{q \in\left(C^{+} \backslash A\left(\nu_{Q_{+}}, \alpha\right)\right) \times\left\{\nu_{Q_{-}}\right\}} \varphi(q, \nu) d_{Q} q \ll \varphi_{E}\left(\nu_{E}\right) e^{-U \alpha^{2}} .
\end{gathered}
$$

If $\nu_{j} \in i[0,1-\varepsilon) \cup\left(0, \nu_{0}\right]$ for some $j \in Q_{+}$, then

$$
\int_{q \in A\left(\nu_{Q_{+}}, \varepsilon\right) \times\left\{\nu_{Q_{-}}\right\}} \varphi(q, \nu) d_{Q} q=0 .
$$

If $A\left(\nu_{Q_{+}}, \alpha\right) \subset C^{+}$, then

$$
\int_{q \in A\left(\nu_{Q_{+}}, \alpha\right) \times\left\{\nu_{Q_{-}}\right\}} \varphi(q, \nu) d_{Q} q=\varphi_{E}\left(\nu_{E}\right)\left(1+O\left(e^{-U \alpha^{2}}\right)\right) .
$$

Proof. We have

$$
\int_{q \in A\left(\nu_{Q_{+}}, \alpha\right) \times\left\{\nu_{Q_{-}}\right\}} \varphi(q, \nu) d_{Q} q=\varphi_{E}\left(\nu_{E}\right) \prod_{j \in Q_{+}} I_{\alpha}^{j}\left(\nu_{j}\right) .
$$

This directly implies (84). If $\left.A \nu_{Q_{+}}, \alpha\right) \subset C^{+}$, then $\nu_{j} \in i[1+\alpha, \infty)$ for any $j \in Q_{+}$. Hence (87) follows. Equality (86) also follows from Lemma 4.3.

For 85) we use

$$
\begin{aligned}
& \int_{q \in\left(C^{+} \backslash A\left(\nu_{Q_{+}}, \alpha\right)\right) \times\left\{\nu_{Q_{-}}\right\}} \varphi(q, \nu) d_{Q} q \\
& \quad \ll \varphi_{E}\left(\nu_{E}\right) \sum_{m \in Q_{+}} J_{\alpha}^{m}\left(\nu_{m}\right) \prod_{j \in Q_{+} \backslash\{m\}}\left(I_{\alpha}^{j}\left(\nu_{j}\right)+J_{\alpha}^{j}\left(\nu_{j}\right)\right) \\
& \quad \ll \varphi_{E}\left(\nu_{E}\right) \sum_{m \in Q_{-}} O\left(e^{-U \alpha^{2}}\right) O(1)^{\left|Q_{+}\right|-1} .
\end{aligned}
$$


4.1.4. Error term. We will use these comparison results to estimate the following difference:

$$
\begin{aligned}
& \tilde{\mathrm{N}}^{r}\left(\varphi_{E} \otimes C\right)-\int_{C} \tilde{\mathrm{N}}^{r}(\varphi(q, \cdot)) d_{Q} q \\
& =\sum_{\varpi, \nu_{\varpi} \in C}\left|c^{r}(\varpi)\right|^{2}\left(\varphi_{E}\left(\nu_{\varpi, E}\right)-\int_{C} \varphi\left(q, \nu_{\varpi}\right) d_{Q} q\right) \\
& \quad-\sum_{\varpi, \nu_{\varpi, Q} \notin C}\left|c^{r}(\varpi)\right|^{2} \int_{C} \varphi\left(q, \nu_{\varpi}\right) d_{Q} q,
\end{aligned}
$$

with $X_{j}$ as in 4.1 .2

We write the difference in (88) as $T_{i}+T_{b}+T_{o}$, given by the respective conditions $\nu_{\varpi, Q_{+}} \in C^{+}(-\varepsilon), \nu_{\varpi, Q_{+}} \in C^{+}[\varepsilon]$, and $\nu_{\varpi, Q_{+}} \notin C^{+}(\varepsilon)$.

4.1.5. Inner error term. $C^{+}(-\varepsilon)$ is contained in the subset

$$
X_{i}=\bigcup_{\nu \in C^{+}(-\varepsilon)} A(\nu, \varepsilon)
$$

of $C^{+}$, which is $(1, \varepsilon)$-blunt. With (87) and Proposition 3.2.

$$
\begin{aligned}
T_{i} & \left.\ll \sum_{\varpi, \nu_{\varpi, Q_{+}} \in C^{+}(-\varepsilon)}|| c^{r}(\varpi)\right|^{2}\left|e^{-U \varepsilon^{2}}\right| \varphi_{E}\left(\nu_{\varpi, E}\right) \mid \\
& \ll{ }_{F, I, r} N_{E}\left(\varphi_{E}\right) \tilde{V}\left(X_{i} \times C^{-}\right) e^{-U \varepsilon^{2}} \leq N_{E}\left(\varphi_{E}\right) e^{-U \varepsilon^{2}} \tilde{V}(C) .
\end{aligned}
$$

4.1.6. Boundary error term. With (84),

$$
T_{b} \ll \sum_{\varpi, \nu_{\varpi, Q_{+}} \in C^{+}[\varepsilon]}\left|c^{r}(\varpi)\right|^{2}\left|\varphi_{E}\left(\nu_{\varpi, E}\right)\right| .
$$

We put $C^{+}[\varepsilon]$ in the $(1, \varepsilon)$-blunt set $\bigcup_{\nu \in C^{+}[\varepsilon]} A(\nu, \varepsilon)$ contained in $C^{+}[2 \varepsilon]$. This leads to

$$
T_{b} \ll_{F, I, r} N_{E}\left(\varphi_{E}\right) \tilde{V}_{1}\left(C^{+}[2 \varepsilon] \times C^{-}\right) .
$$

4.1.7. Outer error term. Now we use (85) and use the fact that the $(1, \varepsilon)$-blunt set

$$
\bigcup_{\nu \in C^{+}(\varepsilon(n+1)) \backslash C^{+}(\varepsilon n)} A(\nu, \varepsilon)
$$

is contained in $C^{+}(\varepsilon(n+2)) \backslash C^{+}(\varepsilon(n-1))$. Then

$$
\begin{aligned}
T_{o} \ll_{F, I, r} & N_{E}\left(\varphi_{E}\right) \\
& \cdot \sum_{n=1}^{\infty} e^{-U \varepsilon^{2} n^{2}}\left(\tilde{V}_{1}\left(C^{+}(\varepsilon(n+2))\right)-\tilde{V}_{1}\left(C^{+}(\varepsilon(n-1))\right)\right) .
\end{aligned}
$$

4.1.8. Growth on shells. Estimate (91) has the disadvantage that the bound is given by an infinite sum. Let us consider $D_{n}=C^{+}(\varepsilon(n+1))-C^{+}(\varepsilon n)$. The size of the sum

$$
\sum_{n=1}^{\infty} e^{-U \varepsilon^{2} n^{2}} \tilde{V}_{1}\left(D_{n-1} \cup D_{n} \cup D_{n+1}\right)
$$

depends mainly on the size of $\tilde{V}\left(D_{n}\right)$ for small values of $n$. 
Lemma 4.5. There is a constant $R=R\left(\left|Q_{+}\right|\right)>1$, not depending on $\varepsilon \in\left(0, e^{-1}\right]$, such that $\tilde{V}_{1}\left(D_{n}\right) \leq R^{n} C^{+}[\varepsilon]$ for any $n \geq 0$.

Proof. The sets $D_{n}$ are subsets of $\left(i[0, \infty) \cup\left(0, \nu_{0}\right]\right)^{Q_{+}}$, which we identify with $\left[-\nu_{0}, \infty\right)^{A_{+}}: q_{j} \in i[0, \infty)$ is replaced by $q_{j} \in[0, \infty)$, and $q_{j} \in\left(0, \nu_{0}\right]$ by $-q_{j}$. Now in each factor the distance dist in (67) is for each coordinate given by the absolute value of the difference. The measure $d_{Q_{+}}$corresponds to the Lebesgue measure on $\mathbb{R}^{Q_{+}}$.

If $q \in D_{n}$, then there is $\nu \in C^{+}$such that dist $\left(q_{j}, \nu_{j}\right) \leq \varepsilon(n+1)$ for all $j$ and $\operatorname{dist}\left(q_{l}, \nu_{l}\right)>\varepsilon n$ for some $l$. For the latter $l$ we define $\tilde{q}_{l} \in\left[-\nu_{0}, \infty\right)$ such that $\operatorname{dist}\left(\tilde{q}_{l}, \nu_{l}\right) \leq \varepsilon n$ and $\operatorname{dist}\left(\tilde{q}_{l}, q_{l}\right)=\varepsilon$. For the other coordinates we put $\tilde{q}_{j}=q_{j}$.

This implies that each point of $D_{n+1}$ can be moved into $D_{n}$ by a translation $T_{\mathbf{v}}$ in $\mathbb{R}^{Q_{+}}$over a vector $\mathbf{v}$ with coordinates in $\{0, \varepsilon,-\varepsilon\}$. Hence

$$
D_{n+1} \subset \bigcup_{\mathbf{v}} T_{\mathbf{v}} D_{n} .
$$

There are $3^{\left|Q_{+}\right|}$translates. For each of these translates

$$
\tilde{V}_{1}\left(T_{\mathbf{v}} D_{n}\right)=\int_{D_{n}} p(x+\mathbf{v}) d_{Q} x
$$

Now we have

$$
p(x+\mathbf{v}) \leq \sum_{m=0}^{\left|Q_{+}\right|}\left(\begin{array}{c}
\left|Q_{+}\right| \\
m
\end{array}\right) \varepsilon^{m} p(x) .
$$

Hence we have $\tilde{V}_{1}\left(T_{\mathbf{v}} D_{n}\right) \leq R_{1} \tilde{V}_{1}\left(D_{n}\right)$, with $R_{1}=\sum_{m=0}^{\left|Q_{+}\right|}\left(\begin{array}{c}\left|Q_{+}\right| \\ m\end{array}\right) e^{-m}$, for any $\varepsilon \in$ $\left(0, e^{-1}\right]$. This implies $\tilde{V}_{1}\left(D_{n+1}\right) \leq R \tilde{V}_{1}\left(D_{n}\right)$ with

$$
R:=R\left(\left|Q_{+}\right|\right)=\left(3\left(1+e^{-1}\right)\right)^{\left|Q_{+}\right|} .
$$

Hence $\tilde{V}_{1}\left(D_{n}\right) \leq R^{n} \tilde{V}_{1}\left(D_{0}\right) \leq R^{n} \tilde{V}_{1}\left(C^{+}[\varepsilon]\right)$.

The factor $3^{\left|Q_{+}\right|}$is much too large in most cases, since the translates $T_{\mathbf{v}} D_{n}$ overlap a lot, and cover more than $D_{n+1}$.

To use this lemma in an estimate of the sum in (91) we assume that $U \varepsilon^{2} \geq D$ with $D:=\log R$. Then $n \mapsto e^{-U \varepsilon^{2} n^{2}} R^{n}$ is a decreasing function, and

$$
\begin{aligned}
\sum_{n=1}^{\infty} e^{-U \varepsilon^{2} n^{2}} & \left(R^{n+1}+R^{n}+R^{n-1}\right) \\
\leq & \left(R^{2}+R+1\right) e^{-U \varepsilon^{2}}+\left(R+1+R^{-1}\right) \int_{x=1}^{\infty} e^{-U \varepsilon^{2} x^{2}} R^{x} d x \\
& \ll_{\left|Q_{+}\right|} e^{-U \varepsilon^{2}}+\frac{e^{-U \varepsilon^{2}+\log C}}{\sqrt{1-\frac{\log C}{2 U \varepsilon^{2}}}} \ll e^{-U \varepsilon^{2}} .
\end{aligned}
$$

Therefore, under the assumption $U \varepsilon^{2} \geq D$, where $D=\log (R)$, the outer error term (91) can be estimated by

$$
O_{\left|Q_{+}\right|}\left(e^{-U \varepsilon^{2}} \tilde{V}_{1}\left(C^{+}[\varepsilon] \times C^{-}\right)\right)
$$

and hence be absorbed into the term $O\left(\tilde{V}_{1}\left(C^{+}[2 \varepsilon] \times C^{-}\right)\right)$. This concludes estimation of the error term; hence the proof of Proposition 4.1 is now complete. 
4.2. Choice of the parameters $U$ and $\varepsilon$. We now arrive at the delicate point where the parameters $U, \varepsilon$ will be linked to the volume quantities, depending on the set $C$. Let us rewrite the error term $E$ in (79):

$$
\begin{gathered}
E=E(C, U, \varepsilon)=\left(e^{t_{0} U\left|Q_{+}\right|} m_{\rho}(C)+e^{-U \varepsilon^{2}}+U^{-1 / 2}+\beta_{\varepsilon}(C)\right) \tilde{V}_{1}(C), \\
m_{\rho}(C)=\frac{\tilde{V}_{\rho}\left(C^{+}\right) \tilde{V}_{-A}\left(C^{-}\right)}{\tilde{V}_{1}(C)}, \quad \beta_{\varepsilon}\left(C^{+}\right)=\frac{\tilde{V}_{1}\left(C^{+}[2 \varepsilon]\right)}{\tilde{V}_{1}\left(C^{+}\right)} .
\end{gathered}
$$

We will require that $m_{\rho}(C)$ and $\beta_{\varepsilon}(C)$ get small so as to be able to control the error term in Proposition 4.1. Furthermore we will need to choose $U, \varepsilon$ suitably. It turns out that a convenient election will be to let $U$ (resp. $\varepsilon$ ) tend slowly to $\infty$ (resp. $0)$ in such a way that $U \varepsilon^{2}$ still tends to $\infty$. Keeping $e^{t_{0} U\left|Q_{+}\right|} m_{\rho}(C)$ and $U^{-1 / 2}$ in mind, we choose

$$
U=U(C)=\frac{1}{t_{0}\left|Q_{+}\right|}\left(\left|\log m_{\rho}(C)\right|-\frac{1}{2} \log \left|\log m_{\rho}(C)\right|\right) .
$$

The condition $U \geq e^{2} D$ with $D=\log R\left(\left|Q_{+}\right|\right)$, as in Proposition 4.1, is satisfied if $m_{\rho}(C)$ is sufficiently small. With this choice,

$$
e^{t_{0} U\left|Q_{+}\right|} m_{\rho}(C)+U^{-1 / 2} \ll\left|\log m_{\rho}(C)\right|^{-1 / 2} .
$$

The contribution $e^{-U \varepsilon^{2}}$ should also be small. We take $\varepsilon$ only slightly larger than $U^{-1 / 2}$ :

$$
\begin{aligned}
\varepsilon & =\varepsilon(C)=\sqrt{\frac{\log \left|\log m_{\rho}(C)\right|}{2 U}} \\
& =\sqrt{\frac{t_{0}\left|Q_{+}\right| \log \left|\log m_{\rho}(C)\right|}{2\left|\log m_{\rho}(C)\right|\left(1-\frac{1}{2} \frac{\log \left|\log m_{\rho}(C)\right|}{\left|\log m_{\rho}(C)\right|}\right.}} .
\end{aligned}
$$

The quantity $\varepsilon$ tends to zero as $m_{\rho}(C)$ tends to zero, and $\varepsilon^{2} U=\frac{\log \left|\log m_{\rho}(C)\right|}{2}$ tends to $\infty$ as $m_{\rho}(C)$ tends to 0 . Thus, $\varepsilon$ satisfies the conditions in Proposition 4.1 for sufficiently small values of $m_{\rho}(C)$.

Let us consider the term $U^{-1 / 2} \tilde{V}_{1}(C)$ in the error term. With the choice of $U$ and $\varepsilon$ just indicated, this term is slightly larger than

$$
\frac{\tilde{V}_{1}(C)}{\left(\log m_{\rho}(C)\right)^{2}}=\frac{\tilde{V}_{1}(C)}{\left(\log \tilde{V}_{1}(C)-\log \left(\tilde{V}_{\rho}\left(C^{+}\right) \tilde{V}_{-A}\left(C^{-}\right)\right)\right)^{2}} .
$$

So the size of the error term will in general differ from the size of the main term by a logarithmic factor. Therefore we now switch from giving $O$-estimates to asymptotic estimates with an $o$-term.

In this way we obtain as the endpoint of the first stage of the derivation of the asymptotic formula:

Theorem 4.6. Let $r \in \mathcal{O}^{\prime} \backslash\{0\}$. Divide up the set of real places of $F$ as $E \sqcup Q_{+} \sqcup Q_{-}$ with $Q=Q_{+} \cup Q_{-} \neq \emptyset$. Let $\mathcal{C}$ be the collection of bounded $d_{Q^{-}}$measurable sets $C=C^{+} \times C_{-}$such that $C^{+} \subset(i[1, \infty))^{Q_{+}}, C^{-} \subset\left[\frac{1}{2}, \infty\right)^{Q_{-}}$. Let $\rho \in(0,1)$ be as in Proposition 4.1, and let $\varepsilon(C)$ be as chosen in (96). 
For each product $\varphi_{E}=\bigotimes_{j \in E} \varphi_{j}$ of local test functions, and for each family $t \mapsto C_{t}$ in $\mathcal{C}$ such that as $t \rightarrow \infty$

$$
\begin{array}{r}
\tilde{V}_{\rho}\left(C_{t}^{+}\right) \tilde{V}_{-A}\left(C_{t}^{-}\right)=o\left(\tilde{V}_{1}\left(C_{t}\right)\right), \\
\text { if } Q_{+} \neq \emptyset, \text { then } \tilde{V}_{1}\left(C_{t}^{+}\left[2 \varepsilon\left(C_{t}^{+}\right)\right]\right) \tilde{V}_{1}\left(C_{t}^{-}\right)=o\left(\tilde{V}_{1}\left(C_{t}\right)\right),
\end{array}
$$

the following asymptotic result holds as $t \rightarrow \infty$ :

$$
\tilde{\mathrm{N}}^{r}\left(\varphi_{E} \otimes C_{t}\right)=\frac{2 \sqrt{\left|D_{F}\right|}}{(2 \pi)^{d}} \operatorname{Pl}\left(\varphi_{E} \otimes C_{t}\right)\left(1+o_{F, I, r}(1)\right) .
$$

For families $t \mapsto C_{t}$ as in the theorem, the quantities $\tilde{V}_{1}\left(C_{t}\right)$ and $\widetilde{\mathrm{Pl}}\left(C_{t}\right)$ have the same size. So we have to replace $o\left(\tilde{V}_{1}\left(C_{t}\right)\right)$ by $\widetilde{\mathrm{Pl}}\left(C_{t}\right)$ in (19).

When formulating the asymptotic formula in $\lambda$-space, the quantity $\tilde{V}_{b}\left(C_{t}\right)$ corresponds to $V_{b}\left(C_{t}\right)$, given by the measure $V_{b}=\bigoplus_{j} V_{b, j}$ :

$$
\begin{aligned}
V_{b, j}(f)=\frac{1}{2} & \int_{5 / 4}^{\infty} f(\lambda)(\lambda-1 / 4)^{(b-1) / 2} d \lambda+\frac{1}{2} \int_{\lambda_{0}}^{5 / 4} f(\lambda) \frac{d \lambda}{\sqrt{\left|\lambda-\frac{1}{4}\right|}} \\
& +\sum_{\beta \in \frac{\xi_{j}+1}{2}+\mathbb{N}_{0}}|\beta|^{b} f\left(\frac{\beta}{2}\left(1-\frac{\beta}{2}\right)\right) .
\end{aligned}
$$

4.3. Unions. It is also useful to state an asymptotic formula for families of disjoint unions $t \mapsto c_{t}=\bigsqcup_{n} C(n)_{t}$, where $C(n)_{t}=C(n)_{t}^{+} \times C(n)_{t}^{-}$, with $n$ in a countable index set. Then we have to replace (94) by

$$
\begin{aligned}
m_{\rho}\left(C_{t}\right) & =\frac{1}{\tilde{V}_{1}\left(C_{t}\right)} \sum_{n} \tilde{V}_{\rho}\left(C(n)_{t}^{+}\right) \tilde{V}_{-A}\left(C(n)_{t}^{-}\right), \\
\beta_{\varepsilon}\left(C_{t}\right) & =\frac{1}{\tilde{V}_{1}\left(C_{t}\right)} \sum_{n} \tilde{V}_{1}\left(C(n)_{t}^{+}[2 \varepsilon]\right) \tilde{V}_{1}\left(C(n)_{t}^{-}\right) .
\end{aligned}
$$

Proceeding with these choices, we obtain:

Proposition 4.7. Let $r \in \mathcal{O}^{\prime} \backslash\{0\}$. Let $E \sqcup Q$ be a partition of the set of real places of $F$, with $Q \neq \emptyset$. Let $t \mapsto C_{t}$ be a family of bounded $d_{Q}$-measurable sets such that for each $t$,

$$
C_{t}=\bigsqcup_{n} C(n)_{t},
$$

with each $C(n)_{t}$ in the collection $\mathcal{C}$ in Theorem 4.6. The decomposition $Q=Q_{n}^{+} \sqcup$ $Q_{n}^{-}$may depend on $n$. Under the conditions

$$
\begin{aligned}
\sum_{n} \tilde{V}_{\rho}\left(C(n)_{t}^{+}\right) \tilde{V}_{-A}\left(C(n)_{t}^{-}\right) & =o\left(\operatorname{Pl}\left(C_{t}\right)\right), \\
\text { if } Q_{+} \neq \emptyset, \text { then } \sum_{n} \tilde{V}_{1}\left(C(n)_{t}^{+}\left[2 \varepsilon\left(C_{t}\right)\right]\right) \tilde{V}_{1}\left(C(n)_{t}^{-}\right) & =o\left(\operatorname{Pl}\left(C_{t}\right)\right),
\end{aligned}
$$


the asymptotic formula (98) holds for each choice of $\varphi_{E}$ as a product of local test functions.

\section{Asymptotic Formula, SeCond stage}

We still have the freedom to choose the test function $\varphi_{E}$. In the second stage we use this freedom to fill in the region $i[0,1) \cup\left(0, \nu_{0}\right]$ for the coordinates of $\nu_{\varpi}$ in $E$. More generally, by specializing $\varphi_{E}$ we can make the asymptotic formula look sharply at the coordinate of $\nu_{\varpi}$ in $E$.

We shall choose the test functions $\varphi_{j}$ with $j \in E$ as approximations of the characteristic functions of "intervals" in $i[0, \infty) \cup(0, \infty)$. Proceeding to a description in terms of the eigenvalue vectors $\lambda_{\varpi}$, we obtain Theorem 5.3, which gives

$$
\mathrm{N}^{r}\left(B \times \hat{C}_{t}^{+} \times \hat{C}_{t}^{-}\right)=\frac{2 \sqrt{\left|D_{F}\right|}}{(2 \pi)^{d}} \operatorname{Pl}\left(B \times \hat{C}_{t}^{+} \times \hat{C}_{t}^{-}\right)(1+o(1)),
$$

where $\hat{C}_{t}^{+}$and $\hat{C}_{t}^{-}$are the sets corresponding to $C_{t}^{+}$and $C_{t}^{-}$under the transformation $\nu \mapsto \lambda$. The set $B$ is a fixed box in $\mathbb{R}^{E}$. This result is strong, but not adequate for some obvious families. Proposition 4.7 gives a generalization allowing us to apply the asymptotic formula to families that are countable disjoint unions of families of the form $t \mapsto B \times \hat{C}_{t}^{+} \times \hat{C}_{t}^{-}$.

5.1. Compactly supported functions at the places in $E$. For a family $t \mapsto C_{t}$ satisfying the conditions in Theorem 4.6 or in Proposition 4.7, we rewrite (98) as follows:

$$
\lim _{t \rightarrow \infty} \frac{2 \sqrt{\left|D_{F}\right|}}{(2 \pi)^{d}} \frac{\tilde{\mathrm{N}}^{r}\left(\varphi_{E} \otimes C_{t}\right)}{\operatorname{Pl}\left(C_{t}\right)}=\widetilde{\mathrm{Pl}}_{E}\left(\varphi_{E}\right) .
$$

Proposition 5.1. Let $r \in \mathcal{O}^{\prime} \backslash\{0\}$ and the decomposition $E \sqcup Q_{+} \sqcup Q_{-}$be as before. For $f_{E}=\bigotimes_{j \in E} f_{j}$ with $f_{j}: \mathbb{R} \rightarrow \mathbb{R}$, define $\tilde{f}_{E}=\bigotimes_{j \in E} \tilde{f}_{j}$ by $\tilde{f}_{j}(\nu)=f_{j}\left(\frac{1}{4}-\nu^{2}\right)$. If $f_{j} \in C_{c}^{1}(\mathbb{R})$ for any $j \in E$, then

$$
\lim _{t \rightarrow \infty} \frac{2 \sqrt{\left|D_{F}\right|}}{(2 \pi)^{d}} \frac{\tilde{\mathrm{N}}^{r}\left(\tilde{f}_{E} \otimes C_{t}\right)}{\operatorname{Pl}\left(C_{t}\right)}=\mathrm{Pl}_{E}\left(f_{E}\right) .
$$

The proof is given in the remainder of this subsection. We can follow the approach in 2] closely.

5.1.1. Functionals. First we formulate two lemmas to be used in the proof.

For $f: \mathbb{R}^{E} \rightarrow \mathbb{C}$ put

$$
A_{t}^{r}(f)=\frac{2 \sqrt{\left|D_{F}\right|}}{(2 \pi)^{d}} \frac{\tilde{\mathrm{N}}^{r}\left(\tilde{f} \otimes C_{t}\right)}{\widetilde{\mathrm{Pl}}\left(C_{t}\right)},
$$

where $\tilde{f}$ is defined by $\tilde{f}(\nu)=f\left(\left(\frac{1}{4}-\nu_{j}^{2}\right)_{j \in Q}\right)$. This defines a measure on $\mathbb{R}^{E}$. We want to compare it to the measure $f \mapsto \widetilde{\mathrm{Pl}}(\tilde{f})=\operatorname{Pl}(f)$.

Lemma 5.2. Let $r \in \mathcal{O}^{\prime} \backslash\{0\}$, let $T \mapsto f_{T}$ be a family of real-valued functions on $\mathbb{R}^{E}$, and let $f$ and $h$ also be real-valued on $\mathbb{R}^{E}$, such that

i) $f, h$ and every $f_{T}$ is integrable for all $A_{t}^{r}$ and for $\mathrm{Pl}$.

ii) $\lim _{t \rightarrow \infty} A_{t}^{r}\left(f_{T}\right)=\operatorname{Pl}\left(f_{T}\right)$ for all $T$.

iii) $\lim _{t \rightarrow \infty} A_{t}^{r}(h)=\mathrm{Pl}(h)$. 
iv) There is a function $T \mapsto a(T)$ such that $a(T)=o(1)$ as $T \rightarrow \infty$, and

$$
\left|f_{T}(x)-f(x)\right| \leq a(T) h(x) \quad \text { for all } x \in \mathbb{R}^{E} .
$$

Then $\lim _{t \rightarrow \infty} A_{t}^{r}(f)=\operatorname{Pl}(f)$.

Proof. The measures $A_{t}^{r}$ are non-negative; hence

$$
A_{t}^{r}\left(f_{T}\right)-a(T) A_{t}^{r}(h) \leq A_{t}^{r}(f) \leq A_{t}^{r}\left(f_{T}\right)+a(T) A_{t}^{r}(h) .
$$

Taking the limit as $t \rightarrow \infty$ of both terms on the side shows that for all $T$,

$$
0 \leq \limsup _{t \rightarrow \infty} A_{t}^{r}(f)-\liminf _{t \rightarrow \infty} A_{t}^{r}(f) \leq 2 a(T) A_{t}^{r}(h) .
$$

Since $a(T)=o(1)$, the $\operatorname{limit}_{t \rightarrow \infty} \lim _{t}^{r}(f)$ exists.

With the non-negativity of $\mathrm{Pl}$ we derive from iv) that for all $T$,

$$
\operatorname{Pl}\left(f_{T}\right)-a(T) \operatorname{Pl}(h) \leq \operatorname{Pl}(f) \leq \operatorname{Pl}\left(f_{T}\right)+a(T) \operatorname{Pl}(h) .
$$

Hence for all $T$,

$$
\left|\operatorname{Pl}(f)-\lim _{t \rightarrow \infty} A_{t}^{r}(f)\right| \leq 2 a(T) \mathrm{Pl}(h) .
$$

This gives the statement of the lemma.

5.1.2. Approximation. Now we start the proof of Proposition 5.1. For given $f_{j} \in$ $C_{c}^{1}(\mathbb{R})$ we take

$$
\varphi_{j}(\nu)=\sqrt{\frac{T}{\pi}} \int_{-\infty}^{\infty} e^{-T\left(\lambda-\frac{1}{4}+\nu^{2}\right)^{2}} f_{j}(\lambda) d \lambda,
$$

with $T$ a large positive parameter. Again we use a Gaussian kernel function, but now in the $\lambda$-space. This defines $\varphi_{j}(\nu)$ as an even holomorphic function of $\nu \in \mathbb{C}$, with exponential decay on the strip $|\operatorname{Re} \nu| \leq \tau$, and for $\nu \in \mathbb{R}$. So $\varphi_{j}$ is a test function in the sense of 2.1 .1 For $\nu \in \mathbb{R} \cup i \mathbb{R}$, it is given by

$$
\varphi_{j}(\nu)=\sqrt{\frac{T}{\pi}} \int_{-\infty}^{\infty} e^{-T \lambda^{2}} f_{j}\left(\lambda+\frac{1}{4}-\nu^{2}\right) d \lambda .
$$

We view $\varphi_{j}$ as an approximation of $\tilde{f}_{j}$. Similarly, $\varphi_{E}$ is an approximation of $\tilde{f}_{E}$.

5.1.3. Local estimates. Since $f_{j}$ is real-valued, we have $\left|\varphi_{j}(\nu)\right| \leq\left\|f_{j}\right\|_{\infty}$ for any $\nu \in \mathbb{R} \cup i \mathbb{R}$. Take $N$ large, such that $\operatorname{Supp} f_{j} \subset[-N, N]$ for any $j \in E$. If $\nu \in \mathbb{R} \cap i \mathbb{R}$ with $|\nu| \geq \sqrt{2 N+1}$, and $-N \leq \lambda \leq N$, then

$$
\lambda-\frac{1}{4}+\nu^{2} \begin{cases}\leq N-\frac{1}{4}-|\nu|^{2} \leq-\frac{1}{2}|\nu|^{2}-\frac{3}{4} & \text { if } \nu \in i \mathbb{R}, \\ \geq-N-\frac{1}{4}+|\nu|^{2} \geq \frac{1}{2}|\nu|^{2}+\frac{1}{4} & \text { if } \nu \in \mathbb{R} .\end{cases}
$$

Hence $e^{-T\left(\lambda-\frac{1}{4}+\nu^{2}\right)^{2}} \leq e^{-\frac{1}{4} T \nu^{4}}$ for such values of $\nu$ and $\lambda$. Together, these facts give for $\nu \in \mathbb{R} \cup i \mathbb{R}$ :

$$
\left|\varphi_{j}(\nu)\right| \leq \begin{cases}\left\|f_{j}\right\|_{\infty} & \text { if }|\nu|<\sqrt{2 N+1} \\ 2 N \sqrt{\frac{T}{\pi}} e^{-\frac{1}{4} T \nu^{4}}\left\|f_{j}\right\|_{\infty} & \text { if }|\nu| \geq \sqrt{2 N+1}\end{cases}
$$

We recall the positive test function $\varphi_{p, E}=\bigotimes_{j \in E} \varphi_{p}$ in (72), which gives for $p>\tau:$

$$
\varphi_{p}\left(\nu_{j}\right)= \begin{cases}\left(p^{2}-\nu_{j}^{2}\right)^{-a / 2} & \text { if }|\operatorname{Re} \nu| \leq \tau \\ \left(p^{2}+\nu_{j}^{2}\right)^{-a / 2} & \text { otherwise }\end{cases}
$$


We take $T \geq T_{0}=4$. For $\nu \in \mathbb{R} \cup i \mathbb{R},|\nu| \geq \sqrt{2 N+1}$, we have $T e^{-\frac{1}{4} T|\nu|^{4}} \leq$ $T_{0} e^{-|\nu|^{4}} \ll|\nu|^{-a}$, and hence

$$
\left|\varphi_{j}(\nu)\right| \ll T^{-1 / 2} N\left\|f_{j}\right\|_{\infty}|\nu|^{-a} \ll_{N} T^{-1 / 2} \varphi_{p}(\nu) .
$$

For $\nu \in \mathbb{R} \cup i \mathbb{R},|\nu| \leq \sqrt{2 N+1}$ :

$$
\begin{aligned}
\varphi_{j}(\nu) & -\tilde{f}_{j}(\nu)=\frac{1}{\sqrt{\pi}} \int_{-\infty}^{\infty} e^{-y^{2}}\left(f_{j}\left(\frac{y}{\sqrt{T}}+\frac{1}{4}-\nu^{2}\right)-f_{j}\left(\frac{1}{4}-\nu^{2}\right)\right) d y \\
& \ll\left\|f_{j}^{\prime}\right\|_{\infty} \int_{0}^{T^{1 / 4}} e^{-y^{2}} \frac{y}{\sqrt{T}} d y+\left\|f_{j}\right\|_{\infty} \int_{T^{1 / 4}}^{\infty} e^{-y^{2}} d y \\
& \ll T^{-1 / 2}\left\|f_{j}^{\prime}\right\|_{\infty}+\frac{\left\|f_{j}\right\|_{\infty} e^{-T}}{T^{1 / 4}} \ll T^{-1 / 2}\left(\left\|f_{j}\right\|_{\infty}+\left\|f_{j}^{\prime}\right\|_{\infty}\right) .
\end{aligned}
$$

Under the assumption $|\nu| \leq \sqrt{2 N+1}, \nu \in \mathbb{R} \cup i \mathbb{R}$, we have $\varphi_{p}(\nu) \gg N^{-a / 2}$. Hence

$$
\varphi_{j}(\nu)-\tilde{f}_{j}(\nu) \ll_{j} T^{-1 / 2} N^{a / 2} \varphi_{p}(\nu) .
$$

5.1.4. Global approximation. We apply the Lemma 5.2 with

$$
\begin{aligned}
f_{T}(\lambda) & =\varphi_{E}(\nu), \quad h(\lambda)=\varphi_{p, E}(\nu), \\
f(\lambda) & =f_{E}(\lambda)=\bigotimes_{j \in E} \tilde{f}_{j}\left(\nu_{j}\right),
\end{aligned}
$$

with $\lambda_{j}=\frac{1}{4}-\nu_{j}^{2}, \nu_{j}= \pm \sqrt{\frac{1}{4}-\lambda_{f}}$. Condition i) is satisfied by continuity. We have ii) and iii) from the assumption that $t \mapsto C_{t}$ is a family for which Theorem 4.6 holds. To check condition iv) we note that if $\nu \in(\mathbb{R} \cup i \mathbb{R})^{E}$, such that $\left|\nu_{j}\right| \leq \sqrt{2 N+1}$ for all $j$, then

$$
\left|\varphi_{E}(\nu)-\tilde{f}_{E}(\nu)\right| \ll_{f} T^{-1 / 2} \varphi_{p, E}(\nu) .
$$

If there is at least one $j \in E$ with $\left|\nu_{j}\right| \geq \sqrt{2 N+1}$, then by (112),

$$
\left|\varphi_{E}(\nu)-\tilde{f}_{E}(\nu)\right|=\left|\varphi_{E}(\nu)\right| \ll_{f} T^{-1 / 2} \varphi_{p, E}(\nu) .
$$

The application of the lemmas in $\$ 5.1 .1$ completes the proof of Proposition 5.1.

5.1.5. Remark. We refrain from extending the asymptotic formula to compactly supported functions on $\mathbb{R}^{E}$ that have no product structure.

5.2. Boxes. Proposition 5.1 works with compactly supported continuous functions with product structure. The last step in stage two is the extension to boxes in $\mathbb{R}^{E}$. We now formulate the asymptotic formula in terms of the coordinate $\lambda$, and use the notation

$$
\hat{C}=\left\{\left(\frac{1}{4}-\nu_{j}^{2}\right)_{j \in Q}: \nu \in C\right\}
$$

for $C \subset(i \mathbb{R} \cup \mathbb{R})^{Q}$.

Theorem 5.3. Let $r \in \mathcal{O}^{\prime} \backslash\{0\}$. Let $E \sqcup Q_{+} \sqcup Q_{-}$be a decomposition of the real places of $F$ with $Q=Q_{+} \cup Q_{-} \neq \emptyset$. Let $t \mapsto C_{t}$ be a family of bounded 
$d_{Q}$-measurable sets in the collection $\mathcal{C}$ in Theorem 4.6 or as considered in Proposition 4.7. In particular we suppose that the conditions in (97) or in (102) hold. Let $B_{E}=\prod_{j \in E}\left[A_{j}, B_{j}\right]$ be such that

$$
A_{j}, B_{j} \notin\left\{\frac{b}{2}\left(1-\frac{b}{2}\right): b>1, b \equiv \xi_{j} \bmod 2\right\} .
$$

Then, as $t \rightarrow \infty$,

$$
\mathrm{N}^{r}\left(B_{E} \times \hat{C}_{t}\right)=\frac{2 \sqrt{\left|D_{F}\right|}}{(2 \pi)^{d}} \mathrm{Pl}\left(B_{E} \times \hat{C}_{t}\right)+o\left(\mathrm{Pl}_{Q}\left(\hat{C}_{t}\right)\right) .
$$

Proof. Let $\chi_{E}$ be the characteristic function of $B_{E}$. It has the form $\chi_{E}=\bigotimes_{j \in E} \chi_{j}$. The local characteristic functions $\chi_{j}$ are integrable for $\mathrm{Pl}_{\xi_{j}}$ and for all $A_{t}^{r}$ as defined in (105).

The conditions in the theorem on the endpoints $A_{j}$ and $B_{j}$ make it possible to find for a given $T \geq 1$ elements $u_{T, j}, U_{T, j} \in C_{c}^{1}(\mathbb{R})$ such that $0 \leq u_{T, j} \leq \chi_{j} \leq U_{T, j}$ on $\mathbb{R}$, and such that

$$
\mathrm{Pl}_{\xi_{j}}\left(U_{T, j}-u_{T, j}\right) \leq \frac{1}{T} .
$$

Put $u_{T}=\bigotimes_{j \in E} u_{T, j}, U_{T}=\bigotimes_{j \in E} U_{T, j}$, and $h=\bigotimes_{J \in E} h_{j}$. The asymptotic formula holds for $h$ and for all $u_{T}$ (Proposition 5.1). We have

$$
\begin{aligned}
& 0 \leq U_{T}(\lambda)-u_{T}(\lambda) \leq \sum_{m \in E}\left(U_{T, m}\left(\lambda_{m}\right)-u_{T}\left(\lambda_{m}\right)\right) \prod_{j \in E \backslash\{m\}} h_{j}\left(\lambda_{j}\right), \\
& 0 \leq \mathrm{Pl}_{E}\left(U_{T}-u_{T}\right) \leq \sum_{m \in E} T^{-1} \prod_{j \in E \backslash\{m\}} \mathrm{Pl}_{\xi_{j}}\left(h_{j}\right)=O\left(T^{-1}\right) .
\end{aligned}
$$

Since the $A_{t}^{r}$ are non-negative measures, we have

$$
\begin{aligned}
A_{t}^{r}\left(u_{T}\right) & \leq A_{t}^{r}\left(B_{E}\right) \leq A_{t}^{r}\left(U_{T}\right), \\
\mathrm{Pl}_{E}\left(u_{T}\right) & \leq \liminf _{t \rightarrow \infty} A_{t}^{r}\left(B_{E}\right) \leq \limsup _{t \rightarrow \infty} A_{t}^{r}\left(B_{E}\right) \leq \mathrm{Pl}_{E}\left(U_{T}\right), \\
& \limsup _{t \rightarrow \infty} A_{t}^{r}\left(B_{E}\right)-\liminf _{t \rightarrow \infty} A_{t}^{r}\left(B_{E}\right)=O\left(T^{-1}\right) .
\end{aligned}
$$

So $\lim _{t \rightarrow \infty} A_{t}^{r}\left(B_{E}\right)$ exists and satisfies

$$
\mathrm{Pl}_{E}\left(u_{T}\right) \leq \lim _{t \rightarrow \infty} A_{t}^{r}\left(B_{E}\right) \leq \mathrm{Pl}_{E}\left(U_{T}\right) .
$$

Again applying (119) we conclude that this limit is equal to $\mathrm{Pl}_{E}\left(B_{E}\right)$. Hence $\mathrm{N}^{r}\left(B_{E} \times \hat{C}_{t}\right)=\frac{2 \sqrt{\left|D_{F}\right|}}{(2 \pi)^{d}} \mathrm{Pl}\left(B_{E} \times \hat{C}_{t}\right)+o\left(\mathrm{Pl}_{Q}\left(\hat{C}_{t}\right)\right)$, which is (117).

\section{Special Families}

Theorem 5.3 describes a large class of families of sets for which the asymptotic formula (117) holds. It has the limitation that the factor $C_{t}$ is a subset of $(i[1, \infty) \cup[1 / 2, \infty))^{Q}$, while the region $i[0,1) \cup\left(0, \nu_{0}\right]$ is treated only in the coordinates in $E$. To avoid technical complications we have chosen not to try to derive an asymptotic formula for a larger class of families of sets, but to apply Theorem 5.3 in a number of special cases. This will suffice to give many applications. 
6.1. Boxes. Directly from Theorem 5.3 we get families of boxes of the type $\tilde{B}_{E} \times C_{t}$ with

$$
\left.\left.C_{t}^{+}=\prod_{j \in Q_{+}} i\left[a_{j}(t), b_{j}\right) t\right)\right], \quad C_{t}^{-}=\prod_{j \in Q_{-}}\left[a_{j}(t), b_{j}(t)\right],
$$

where for all $t$

$$
1 \leq a_{j}(t) \leq b_{j}(t) \quad \text { if } j \in Q_{+}, \quad \frac{1}{2} \leq a_{j}(t) \leq b_{j}(t) \quad \text { if } j \in Q_{-} .
$$

A computation of the quantities in (94) shows that

$$
\begin{aligned}
& m_{\rho}\left(C_{t}\right) \ll \prod_{j \in Q_{+}} b_{j}(t)^{\rho-1} \prod_{j \in Q_{-}} \frac{1}{\tilde{V}_{1}\left(C_{t}^{-}\right)}, \\
& \beta_{\varepsilon}\left(C_{t}^{+}\right) \ll \varepsilon \sum_{m \in Q_{+}} \frac{b_{m}(t)}{\left(a_{m}(t)+b_{m}(t)\right)\left(b_{m}(t)-a_{m}(t)+\varepsilon\right)} .
\end{aligned}
$$

In the uninteresting case when $C_{t}^{-}$does not intersect $\prod_{j \in Q_{-}}\left(\frac{\xi_{1}+1}{2}+\mathbb{N}_{0}\right)$, we have $\tilde{\mathrm{N}}^{r}\left(B_{E} \times C_{t}\right)=\widetilde{\mathrm{Pl}}\left(B_{E} \times C_{t}\right)=0$. So we assume that $Q_{-}=\emptyset$ or $\tilde{V}_{1}\left(C_{t}^{-}\right)>0$. Then $\tilde{V}_{1}\left(C_{t}^{-}\right) \geq 1$ if $Q_{-} \neq \emptyset$.

The conclusion is that $m_{\rho}\left(C_{t}\right) \downarrow 0$ as soon as for at least one $j \in Q$ we have $b_{j}(t) \rightarrow \infty$.

With $\varepsilon=\varepsilon\left(C_{t}\right)$ as in (96) it suffices to require in (122) that for any $m \in Q_{+}$,

$$
\left(b_{m}(t)-a_{m}(t)+\varepsilon\right)=o(1) .
$$

This can be achieved by requiring that $b_{j}(t)-a_{j}(t) \geq \gamma\left|\log m_{\rho}\left(C_{t}\right)\right|$ for any $j \in Q_{+}$ and all $t$ large, for any $\alpha \in\left(0, \frac{1}{2}\right)$ and any $\gamma>0$. Thus we have:

Proposition 6.1. The asymptotic formula holds for a family of boxes $t \mapsto \tilde{\Omega}=$ $\tilde{B}_{E} \times C_{t}$ with $B_{E}$ any box in $\mathbb{R}^{E}$ satisfying (116) and $C_{t}$ as in (120) and (121) under the conditions

a) $b_{j}(t) \rightarrow \infty$ for some $j \in Q$.

b) $b_{j}(t)-a_{j}(t) \geq \sigma(t)$ for any $j \in Q_{+}$and all $t$, with

$$
\begin{aligned}
& \sigma(t)=\gamma\left((1-\rho) \sum_{j \in Q_{+}} \log b_{j}(t)+\log \tilde{V}_{1}\left(C_{t}^{-}\right)\right)^{-\alpha} \quad\left(\gamma>0,0<\alpha<\frac{1}{2}\right) . \\
& \text { c) }\left[a_{j}(t), b_{j}(t)\right] \cap\left(\frac{\xi_{j}+1}{2}+\mathbb{N}_{0}\right) \neq \emptyset \text { for all } t \text { and for any } j \in Q_{-} .
\end{aligned}
$$

This proposition implies Theorem [1.3, and its consequences Propositions 1.1 and 1.2 ,

For boxes in the $\lambda$-parameter we have the following result:

Proposition 6.2. The asymptotic formula holds for families of boxes $t \mapsto \Omega_{t}$, where

satisfies the following conditions:

$$
\Omega_{t}=\prod_{j}\left[A_{j}(t), B_{j}(t)\right]
$$

a) If for a fixed $j A_{j}$ and $B_{j}$ are constant, then $A_{j}=A_{j}(t)$ and $B_{j}=B_{j}(t)$ satisfy condition (116).

b) If $A_{j}(t)$ is not constant, then $A_{j}(t) \leq 0$ for all $t$, or $A_{j}(t) \geq \frac{5}{4}$ for all $t$. Similarly for $B_{j}(t)$. 
c) There is a constant $\sigma>0$ such that if $B_{j}(t) \geq \frac{5}{4}$, then

$$
B_{j}(t)-\max \left(A_{j}(t), \frac{5}{4}\right) \geq \sigma\left(\sqrt{\mid B_{j}(t)}+\sqrt{\max \left(A_{j}(t), \frac{5}{4}\right)}\right) .
$$

d) If $A_{j}(t) \leq 0$, then the interval $\left[A_{j}(t), B_{j}(t)\right]$ intersects for all $t$ the set of $\frac{b}{2}\left(1-\frac{b}{2}\right), b>1, b \equiv \xi_{j} \bmod 2$ non-trivially for all $t$. (This intersection may depend on $t$.)

e) $\lim _{t \rightarrow \infty} A_{j}(t)=-\infty$ or $\lim _{t \rightarrow \infty} B_{j}(t)=\infty$ for at least one $j$.

This is not the most general statement for boxes. We have decided not to complicate the proposition by considering non-constant endpoints that have values in $\left(0, \frac{5}{4}\right)$.

Proof. Let $E_{0}$ be the set of places for which $A_{j}$ and $B_{j}$ are constant. We consider partitions $Q_{+} \sqcup Q_{0} \sqcup Q_{-}$of the remaining infinite places of $F$. For each of these partitions $P$ we form

$$
\Omega_{t}^{P}=\Omega_{t} \cap\left(\mathbb{R}^{E_{0}} \cup(i[1, \infty))^{Q_{+}} \cup\left(i[0,1) \cup\left(0, \nu_{0}\right]\right)^{Q_{0}} \cup\left[\frac{1}{2}, \infty\right)^{Q_{-}}\right) .
$$

Suppose $Q_{+} \neq \emptyset$. For $j \in Q_{+}$we write $A_{j}(t)=\max \left(A_{j}(t), \frac{5}{4}\right)$. In the $\nu$ description, the factor $\Omega_{t, j}^{P}$ is of the form $i\left[a_{j}(t), b_{j}(t)\right]$ with $a_{j}(t)=\sqrt{A_{j}(t)-\frac{1}{4}}$ and $b_{j}(t)=\sqrt{B_{j}(t)-\frac{1}{4}}$. Condition c) implies that condition b) in Proposition 6.1 is satisfied.

If $Q_{-} \neq \emptyset$ for $P$, then condition d) implies condition c) in Proposition 6.1.

For the partition $P$ we take $E=E_{0} \cup Q_{0}$, and try to apply Proposition 6.1 to $t \mapsto \Omega_{t}^{P}$. This gives the asymptotic formula for $\Omega_{t}^{P}$, provided either there is $j \in Q_{+}$ for which $B_{j}(t) \rightarrow \infty$ or there is $j \in Q_{-}$for which $A_{j}(t) \rightarrow-\infty$. Otherwise, the set $\Omega_{t}^{P}$ is bounded.

Condition e) implies that the asymptotic formula holds for at least some partition $P$. Thus $\tilde{V}_{1}\left(\Omega_{t}^{P}\right) \rightarrow \infty$ for such $P$. Adding the corresponding finitely many terms we get the asymptotic formula for the union of the $\Omega_{t}^{P}$. For the remaining partitions $P$, the set $\Omega_{t}^{P}$ stays bounded. Adding the corresponding terms to the asymptotic formula does no harm. This gives the asymptotic formula for $t \mapsto \Omega_{t}$.

Now an approximation of $\mathrm{Pl}\left([-X, X]^{d}\right)$ gives Proposition 1.6 .

6.2. Simplices. The results in [2] are for sets of the form

$$
\hat{C}_{t}=\left\{\lambda \in[0, \infty)^{Q_{+}} \times(-\infty, 0)^{Q_{-}}: \sum_{j \in Q}\left|\lambda_{j}\right| \leq t\right\} .
$$

By showing that the asymptotic formula holds for sets of this form, we extend the results in 2 to general character $\chi$ and general compatible central character given by $\xi$.

Proposition 6.3. Let $E \sqcup Q_{+} \sqcup Q_{-}$be a partition of the infinite places of $F$ with $Q=Q_{+} \cup Q_{-} \neq \emptyset$. Let $B_{E}$ be any box in $\mathbb{R}^{E}$ satisfying (116). The asymptotic formula holds for $t \mapsto B_{E} \times \hat{C}_{t}$, and

$$
\operatorname{Pl}\left(\hat{C}_{t}\right) \sim \frac{1}{|Q| !} t^{|Q|} \quad(t \rightarrow \infty) .
$$


Proof. Let us first consider

$$
W_{n}(Y)=\left\{\lambda \in\left[\frac{5}{4}, \infty\right)^{n}: \sum_{j} \lambda_{j} \leq Y\right\} .
$$

We have $V_{1}\left(W_{1}(Y)\right)=\frac{1}{2}\left(Y-\frac{5}{4}\right)$ for $Y \geq \frac{5}{4}$. From

$$
V_{1}\left(W_{n}(Y)\right)=\frac{1}{2} \int_{5 / 4}^{Y} V_{1}\left(W_{n-1}(Y-\lambda)\right) d \lambda,
$$

we obtain by induction that

$$
V_{1}\left(W_{n}(Y)\right)=\frac{1}{2^{n} n !}\left(Y-\frac{5}{4} n\right)_{+}^{n} .
$$

We use $(x)_{+}=0$ if $x<0$, and $(x)_{+}=x$ if $x \geq 0$.

For $\rho \in(0,1)$ we find by the inclusion $W_{n}(Y) \subset[5 / 4, Y]^{n}$ that

$$
V_{\rho}\left(W_{n}(Y)\right)=O_{n}\left(Y^{n(\rho+1) / 2}\right) .
$$

Furthermore, for $\varepsilon$ small in comparison with $Y$, the part of $W_{n}(Y)[2 \varepsilon]$ on which $\lambda_{j}>\frac{5}{4}$ for all $j \in Q_{+}$is contained in $W_{n}\left(Y_{+}\right)-W_{n}\left(Y_{-}\right)$with $Y_{+}=Y+4 \varepsilon n Y^{1 / 2}+$ $4 \varepsilon^{2} n$ and $Y_{-}=Y-4 \varepsilon n Y^{1 / 2}$. The other parts of $W_{n}(Y)[2 \varepsilon]$ are contained in boxes of the form $\left[\frac{5}{4}-2 \varepsilon, Y+2 \varepsilon\right]^{n-1} \times\left[\frac{5}{4}-2 \varepsilon, \frac{5}{4}+\varepsilon\right]$. Hence

$$
\begin{aligned}
& \tilde{V}_{1}\left(\tilde{W}_{n}(Y)[2 \varepsilon]\right) \leq V_{1}\left(W_{n}\left(Y_{+}\right)\right)-V_{1}\left(W_{n}\left(Y_{-}\right)\right)+n O\left(Y^{n-1} \varepsilon\right) \\
& \leq \frac{1}{2^{n} n !}\left(\left(Y+4 \varepsilon n Y^{1 / 2}+4 \varepsilon^{2} n-\frac{5}{4} n\right)^{n}-\left(Y-4 \varepsilon n Y^{1 / 2}-\frac{5}{4} n\right)^{n}\right) \\
& \quad+O_{n}\left(\varepsilon Y^{n-1}\right) \\
& \quad \ll_{n} \varepsilon Y^{n-\frac{1}{2}} .
\end{aligned}
$$

If $Y-\frac{5}{4} n$ is small, we get at least $O(\varepsilon)$, which is $O\left(\varepsilon Y^{n-1 / 2}\right)$ as well.

Now we apply Proposition 4.7 to the following subset of $\hat{C}_{t}$ :

$$
W_{t}=\bigsqcup_{\mathbf{p}} W_{\left|Q_{+}\right|}\left(t-\sum_{j \in Q_{-}}\left(\mathbf{p}_{j}^{2}-\frac{1}{4}\right)\right) \times\{\mathbf{p}\},
$$

where $\mathbf{p}$ runs over $\prod_{j \in Q_{-}}\left(\frac{3-\xi_{j}}{2}+\mathbb{N}_{0}\right)$ for which $\sum_{j \in Q_{-}}\left(\mathbf{p}_{j}^{2}-\frac{1}{4}\right) \leq t$. For each given $t$, this is a finite union. But as a family depending on $t$ it is an infinite union. We obtain

$$
\begin{aligned}
& V_{1}\left(W_{t}\right)=\sum_{\mathbf{p}} \frac{1}{2^{\left|Q_{+}\right|}\left|Q_{+}\right| !}\left(t-\frac{5}{4}\left|Q_{+}\right|+\frac{1}{4}\left|Q_{-}\right|-\sum_{j \in Q_{-}} \mathbf{p}_{j}^{2}\right)_{+}^{\left|Q_{+}\right|} \prod_{j \in Q_{-}} \mathbf{p}_{j}, \\
& m_{\rho}\left(W_{t}\right) V_{1}\left(W_{t}\right) \ll_{Q_{+}} \sum_{\mathbf{p}}\left(t+\frac{1}{4}\left|Q_{-}\right|-\sum_{j \in Q_{-}} \mathbf{p}_{j}^{2}\right)^{\left|Q_{+}\right|(\rho+1) / 2} \prod_{j \in Q_{-}} \mathbf{p}_{j}^{-A}, \\
& \beta_{\varepsilon}\left(W_{t}\right) V_{1}\left(W_{t}\right) \ll_{Q_{+}} \varepsilon \sum_{\mathbf{p}}\left(t+\frac{1}{4}\left|Q_{-}\right|-\sum_{j \in Q_{-}} \mathbf{p}_{j}^{2}\right)^{\left|Q_{+}\right|-\frac{1}{2}} .
\end{aligned}
$$


We compare the sum for $V_{1}\left(W_{t}\right)$ with the integral

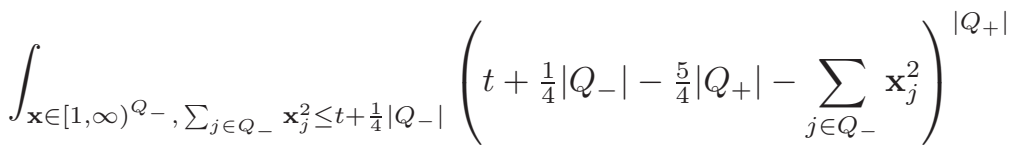

$$
\begin{aligned}
& \cdot \prod_{j \in Q_{-}} \mathbf{x}_{j} d \mathbf{x} \\
& =\int_{\mathbf{y} \in[5 / 4, \infty)^{Q_{-}}, \sum_{j \in Q_{-}} \mathbf{y}_{j} \leq t}\left(t-\frac{5}{4}\left|Q_{+}\right|-\sum_{j \in Q_{-}} \mathbf{y}_{j}\right)^{\left|Q_{+}\right|} \\
& \cdot 2^{-\left|Q_{-}\right|} d \mathbf{y} \\
& =\frac{1}{2^{|Q|}|Q| !}\left(t-\frac{5}{4}|Q|\right)^{|Q|} \sim \frac{t^{|Q|}}{2^{|Q|}|Q| !} .
\end{aligned}
$$

The transition from sum to integral gives a contribution $O\left(t^{|Q|-1}\right)$.

The other sums can be treated similarly. This leads to the estimates $m_{\rho}\left(W_{t}\right) \ll$ $t^{\left|Q_{+}\right|(\rho-1) / 2}$ and $\beta_{\varepsilon}\left(W_{t}\right) \ll t^{-1 / 2}$. This implies that the asymptotic formula holds for $t \mapsto B_{E} \times W_{t}$.

The definition of $\widetilde{\mathrm{P}}$ shows that $\mathrm{Pl}\left(W_{t}\right) \sim \frac{2^{|Q|}}{2^{|Q|}|Q| !} V_{1}\left(W_{t}\right)$.

The remaining parts in $\hat{C}_{t} \backslash W_{t}$ are contained in unions of sets of the form $Y=\left[0, \frac{5}{4}\right] \times[-t, t]^{|Q|-1}$ for which $V_{1}(Y) \ll t^{|Q|-1}$ and $\mathrm{Pl}(Y) \ll t^{|Q|-1}$. So adding these parts does not influence the asymptotic formula or the asymptotic behavior of $\operatorname{Pl}\left(\hat{C}_{t}\right)$.

Proposition 1.7 is a corollary of this result.

6.3. Sectors. Under the assumption $\left|Q_{+}\right|=2$ we consider

$$
S_{p, q, \alpha, t}=\left\{\left(\lambda_{1}, \lambda_{2}\right) \in\left(\frac{5}{4}, \infty\right)^{2}: t \leq \lambda_{1} \leq t+t^{\alpha}, p \lambda_{1} \leq \lambda_{2} \leq q \lambda_{2}\right\},
$$

with $0<p<q, \alpha \leq 1$, and $t \rightarrow \infty$.

Lemma 6.4. For $c \leq 1$,

$$
V_{c}\left(S_{p, q, \alpha, t}\right) \sim \frac{1}{2(c+1)}\left(q^{\frac{c+1}{2}}-p^{\frac{1+c}{2}}\right) t^{c+\alpha} .
$$

Furthermore, for $\varepsilon>0$ small in comparison to $t$ as $t \rightarrow \infty$,

$$
\begin{aligned}
V_{\rho}\left(S_{p, q, \alpha, t}\right) & \ll_{p, q} t^{\rho-1} V_{1}\left(S_{p, q, \alpha, t}\right), \\
V_{1}\left(\tilde{S}_{p, q, \alpha, t}[2 \varepsilon]\right) & \ll_{p, q} \varepsilon t^{\max \left(\frac{3}{2}, \alpha+\frac{1}{2}\right)} V_{1}\left(S_{p, q, \alpha, t}\right) .
\end{aligned}
$$

Proof. (128) is obtained by direct computation based on (99). It immediately implies (129).

The description of $S_{p, q, \alpha, t}$ in $\nu$-space is as follows:

$$
\tilde{S}_{p, q, \alpha, t}=\left\{i\left(t_{1}, t_{2}\right): a \leq t_{1} \leq b, \sqrt{p t_{1}^{2}+\frac{p-1}{4}} \leq t_{2} \leq \sqrt{q t_{1}^{2}+\frac{q-1}{4}}\right\},
$$

with $a=\sqrt{t-\frac{1}{4}}, b=\sqrt{t+t^{\alpha}-\frac{1}{4}}$. The left hand side of $\tilde{S}_{p, q, \alpha, t}[2 \varepsilon]$ is contained in

$$
[a-2 \varepsilon, a+2 \varepsilon] \times[\sqrt{p t-1 / 4}+2 \varepsilon, \sqrt{q t-1 / 4}-2 \varepsilon] .
$$


Its contribution to $\tilde{V}_{1}\left(\tilde{S}_{p, q, \alpha, t}[2 \varepsilon]\right)$ is $O_{q-p}(\varepsilon a \cdot t)=O\left(t^{3 / 2}\right)$. The contribution of the right hand side of $\tilde{S}_{p, q, \alpha, t}[2 \varepsilon]$ has the same order.

On the lower side remains a piece for which $\tilde{V}_{1}$ can be estimated by

$$
\int_{t_{1}=a}^{b} t_{1} \int_{t_{2}=\sqrt{p t_{1}^{2}+\frac{p-1}{4}}-2 \varepsilon(1+p)}^{\sqrt{p t_{1}^{2}+\frac{p-1}{4}}+2 \varepsilon(1+p)} t_{2} d t_{2} d t_{2}
$$

The inner integral can be estimated by $O_{p}\left(\varepsilon \sqrt{p t_{1}^{2}+\frac{p-1}{4}}\right)$. This gives for the total integral

$$
\begin{aligned}
& \ll_{p} \varepsilon\left(\left(p b^{2}+\frac{p-1}{4}\right)^{3 / 2}-\left(p a^{2}+\frac{p-1}{4}\right)^{3 / 2}\right) \\
& \ll_{p} \varepsilon\left(p b^{2}-p a^{2}\right)\left(p b^{2}+\frac{p-1}{4}\right)^{1 / 2} \ll_{p} \varepsilon t^{\alpha+\frac{1}{2}} .
\end{aligned}
$$

Similarly, the upper part contributes $O_{q}\left(\varepsilon t^{\alpha+1 / 2}\right)$.

This lemma shows that if $\alpha \geq \frac{1}{2}$, the asymptotic formula holds for the family $t \mapsto B_{E} \times S_{p, q, \alpha, t}$ for any choice of the box $B_{E}$ as before, where $E$ contains all infinite places except the two places we put in $Q_{+}$. In particular, we obtain Proposition 1.8

6.4. Spheres. We consider the sphere $S_{Q_{+}}(\mathbf{m}, r) \subset(i[1, \infty))^{Q_{-}}$with radius $r$ and center m: The set of $\nu$ with $\sum_{j \in Q_{+}}\left(\left|\nu_{j}\right|-\left|\mathbf{m}_{j}\right|\right)^{2} \leq r^{2}$. We suppose that $\left|\mathbf{m}_{j}\right|-r \geq$ 1 for any $j \in Q_{+}$and that $\left|\mathbf{m}_{j}\right| \rightarrow \infty$ for at least one $j \in \mathbb{Q}_{+}$. A computation by induction on $\left|Q_{+}\right|$leads to

$$
\tilde{V}_{1}\left(S_{Q_{+}}(\mathbf{m}, r)\right)=2 v_{\mid Q_{+}} r^{\left|Q_{+}\right|} \prod_{j \in Q_{+}}\left|\mathbf{m}_{j}\right|,
$$

where $v_{n}$ is the volume of the unit sphere in $\mathbb{R}^{n}$.

Furthermore

$$
\begin{aligned}
\tilde{V}_{\rho}\left(S_{Q_{+}}(\mathbf{m}, r)\right) & \ll r^{\left|Q_{+}\right|} \prod_{j \in Q_{+}}\left|\mathbf{m}_{j}\right|^{\rho}, \\
\tilde{V}_{1}\left(S_{Q_{+}}(\mathbf{m}, r)[2 \varepsilon]\right) & \ll \varepsilon r^{n-1} \prod_{j \in Q_{+}}\left|\mathbf{m}_{j}\right| .
\end{aligned}
$$

These estimates follow from the inclusion

$$
\begin{aligned}
S_{Q_{+}}(\mathbf{m}, r) & \subset \prod_{j \in Q_{+}} i\left[\left|\mathbf{m}_{j}\right|-r,\left|\mathbf{m}_{j}\right|+r\right], \\
S_{Q_{+}}(\mathbf{m}, r)[2 \varepsilon] & \subset S_{Q_{+}}(\mathbf{m}, r+2 \varepsilon \sqrt{n}) \backslash S_{Q_{+}}(\mathbf{m}, r-2 \varepsilon \sqrt{n}) .
\end{aligned}
$$

For the latter inclusion we use the fact that if $\nu$ is on the boundary of $S_{Q_{+}}(\mathbf{m}, r)$, then $\sum_{j \in \mathbf{Q}_{+}}\left(\left|\nu_{j}\right|+2 \varepsilon\right)^{2} \leq r^{2}+4 \varepsilon \sum_{j \in Q_{+}}\left|\nu_{j}\right|+4 \varepsilon^{2} n \leq r^{2}+4 \varepsilon r \sqrt{n}+4 \varepsilon^{2} n$, and similarly $\sum_{j \in \mathbf{Q}_{+}}\left(\left|\nu_{j}\right|-2 \varepsilon\right)^{2} \geq(r-2 \varepsilon \sqrt{n})^{2}$.

These estimates show that the asymptotic formula holds for families $\mathbf{m} \mapsto B_{E} \times$ $S_{Q_{+}}(\mathbf{m}, r)$, where $B_{E}$ is a box as earlier and $Q_{-}=\emptyset$. It works for constant radius $r$, or even for $r$ going down as a multiple of $\left(\log \prod_{j \in Q_{+}}\left|\mathbf{m}_{j}\right|\right)^{-\alpha}$ with $\alpha<\frac{1}{2}$. As a special case we obtain Proposition [1.4. There we have required that all $\mathbf{m}_{j}$ go to infinity, in order to have $\widetilde{\mathrm{Pl}}\left(S_{Q_{+}}(\mathbf{m}, r)\right) \sim 2^{d} \tilde{V}_{1}\left(S_{Q_{+}}(\mathbf{m}, r)\right)$. 
6.5. Slanted strips. Finally we consider, in the case $d=2$, a strip of the form

$$
\tilde{\Omega}_{t}=\{i(x, y): t \leq x \leq 2 t, a x+b \leq y \leq a x+c\},
$$

where $a, b, c \in \mathbb{R}, a>0, c>b$, fixed. The parameter $t$ tends to infinity. We take it such that $\tilde{\Omega}_{t}$ is contained in $(i[1, \infty))^{2}$. Computations similar to those carried out before give

$$
\begin{aligned}
\tilde{V}_{1}\left(\tilde{\Omega}_{t}\right) & \sim \frac{7}{3} a(c-b) t^{3}, \\
\tilde{V}_{\rho}\left(\tilde{\Omega}_{t}\right) & \ll \int_{t}^{2 t} x^{\rho}(c-b)(a x+c)^{\rho} d x \ll a^{\rho}(c-b) t^{2 \rho+1}, \\
\tilde{V}_{1}\left(\tilde{\Omega}_{t}[2 \varepsilon]\right) & \ll \varepsilon t(2 a t+b+c)(b-c+4 \varepsilon)+\int_{t}^{2 t} x \varepsilon(a x+c) d x \\
& \ll{ }_{a, b, c} \varepsilon t^{2}+\varepsilon t^{3} \ll \varepsilon t^{3} .
\end{aligned}
$$

We conclude that the asymptotic formula holds for $\tilde{\Omega}_{t}$, and we thus obtain Proposition 1.5

\section{REFERENCES}

[1] R.W. Bruggeman, Fourier coefficients of cusp forms, Inv. Math. 45 (1978) 1-18 MR0472701 $(57: 12394)$

[2] R.W. Bruggeman, R.J. Miatello, I. Pacharoni, Density results for automorphic forms on Hilbert modular groups, Geometric and Functional Analysis 13 (2003) 681-719 MR 2006554 (2004k:11066)

[3] R.W. Bruggeman, R.J. Miatello, Sum formula for $\mathrm{SL}_{2}$ over a totally real number field, Memoirs Amer. Math. Soc. 197 (2009) no. 919 MR2489364

[4] R.W. Bruggeman, R.J. Miatello, Distribution of square integrable automorphic forms on Hilbert modular groups, Geometry, analysis and topology of discrete groups, 19-39, Adv. Lect. Math. (ALM), 6, Int. Press, Somerville, MA, 2008. MR2464392

[5] H. Donnelly, On the cuspidal spectrum for finite volume symmetric spaces, J. Diff. Geometry 17 (1982) 239-253 MR664496 (83m:58079)

[6] J.J. Duistermaat, J.A.C. Kolk, V.S. Varadajan, Spectra of Compact locally Symmetric Manifolds of Negative Curvature, Inv. Math. 52 (1979) 27-93 MR.532745 (82a:58050a)

[7] I.Y. Efrat, The Selberg trace formula for $\mathrm{PSL}_{2}(\mathbb{R})^{n}$, Memoirs Amer. Math. Soc. 359 (1987) 1-110 MR874084(88e:11041)

[8] D.A. Hejhal, The Selberg trace formula for PSL(2, $\mathbb{R})$, Lect. Notes in Math. 1001, SpringerVerlag, 1983 MR.711197 (86e:11040)

[9] J. Huntley, Spectral multiplicity on products of hyperbolic spaces, Proc. AMS 111 (1991) 1-12 MR.1031667 (91d:11055)

[10] J. Huntley, D. Tepper, A local Weyl's law, the angular distribution and multiplicity of cusp forms on product spaces, Trans. AMS 330 (1992) 97-110 MR1053114 (92f:11071)

[11] A. Ivić, M. Jutila, On the moment of Hecke series at central points. II, Funct. Approx. Comment. Math. 31 (2003) 93-108 MR2059539 (2005g:11076)

[12] M. Jutila, Y. Motohashi, Uniform bounds for Hecke $L$-functions, Acta Math. 195 (2005) 61-115 MR2233686(2007d:11051)

[13] H.H. Kim, F. Shahidi, Cuspidality of symmetric powers with applications, Duke Math. J. 112 (2002) 177-197 MR:1890650(2003a:11057)

[14] J.P. Labesse, W. Müller, Weak Weyl's law for congruence subgroups, Asian J. Math. 8 (2004) 733-746 MR2127945 (2006a:11064)

[15] S. Lang, $\mathrm{SL}_{2}(\mathbb{R})$, Addison-Wesley, 1975 MR0430163 (55:3170)

[16] E. Lapid, W. Müller, Spectral asymptotic for arithmetic quotients of $\mathrm{SL}(n, \mathbb{R}) / \mathrm{SO}(n)$, arXiv:0711.2925v1 [math.RT]

[17] E. Lindenstrauss, A. Venkatesh, Existence and Weyl's law for spherical cusp forms, Geom. Funct. Anal. 17 (2007) 220-251 MR2306657[(2008c:22016) 
[18] Lizhen Ji, The Weyl upper bound on the discrete spectrum of locally symmetric spaces, J. Diff. Geometry 51 (199) 97-147 MR1703605(2001a:11087)

[19] S.D. Miller, On the existence and temperedness of cusp forms for $\mathrm{SL}_{3}(\mathbb{Z})$, J. Reine Angew. Math. 533 (2001) 127-169 MR1823867(2002b:11070)

[20] W. Müller, Weyl's law for the cuspidal spectrum of $\mathrm{SL}_{n}$, C.R. Acad. Sci. Paris 338 (5) (2004) 347-352 MR2057162 (2005a:22006)

[21] A. Selberg, Harmonic analysis and discontinuous groups in weakly symmetric spaces with applications to Dirichlet series, Jour. Indian Math. Soc. (N.S.) 20 (1956) 47-87 MR 0088511 $(19: 531 \mathrm{~g})$

Mathematisch Instituut, Universiteit Utrecht, Postbus 80010, NL-3508 TA Utrecht, NEDERLAND

E-mail address: bruggeman@math.uu.nl

FaMAF-Ciem, Universidad Nacional de Córdoba, Córdoba 5000, Argentina

E-mail address: miatello@mate.uncor.edu 